\title{
Optimal Power Allocation for Jammer Nodes in Wireless Localization Systems
}

\author{
Suat Bayram ${ }^{(1)}$, Musa Furkan Keskin ${ }^{(1)}$, Sinan Gezici ${ }^{(1)}$, Senior Member, IEEE, and Orhan Arikan ${ }^{(1)}$
}

\begin{abstract}
In this paper, optimal power allocation strategies are investigated for jammer nodes in a wireless localization system. Building upon the concept of the restricted Bayesian approach, a generalized optimization strategy, called the restricted scheme, is proposed for power allocation of jammer nodes, and its theoretical properties are characterized. In the restricted scheme, the aim is to maximize the average Cramér-Rao lower bound (CRLB) of target nodes while keeping their minimum CRLB above a predefined level in the presence of average (total) and peak power constraints. It is proved that the average CRLB achieved by the restricted scheme is a strictly decreasing and concave function of the constraint on the minimum CRLB level. A closed-form solution is obtained for the restricted scheme when the tradeoff parameter and the total power limit are below certain thresholds. In addition, it is shown that the optimal solution of the restricted scheme corresponds to the use of at most $N_{T}$ jammer nodes, where $N_{T}$ is the number of target nodes, and that the optimal solution of the minimum CRLB maximization scheme is determined by at most $N_{J}$ target nodes, where $N_{J}$ is the number of jammer nodes. Extensions of the restricted scheme and an alternative scheme that aims to maximize the number of disabled target nodes (whose CRLBs are above a preset level) are considered, and the corresponding optimal strategies for jammer power allocation are identified. Numerical examples are provided to verify the theoretical derivations for various network configurations.
\end{abstract}

Index Terms-Localization, jammer, restricted scheme, power allocation, Cramér-Rao lower bound.

\section{INTRODUCTION}

\section{A. Background and Motivation}

W IRELESS positioning has attracted a significant amount of interest due to its crucial role in numerous applications for location-based services, such as package tracking, home automation, intelligent transport systems, monitoring of patients, and search-and-rescue operations [1]-[4]. Indoor wireless localization systems provide a promising alternative for positioning in environments where GPS signals cannot be uti-

Manuscript received February 15, 2017; revised June 20, 2017 and August 7, 2017; accepted September 11, 2017. Date of publication September 28, 2017; date of current version October 27, 2017. The associate editor coordinating the review of this manuscript and approving it for publication was Dr. Itsik Bergel. (Corresponding author: Sinan Gezici.)

S. Bayram is with Suat Bayram Muhendislik Hizmetleri, Ankara 06760, Turkey (e-mail: suat.bayram@paneratech.com).

M. F. Keskin, S. Gezici, and O. Arikan are with the Department of Electrical and Electronics Engineering, Bilkent University, Ankara 06800, Turkey (e-mail: keskin@ee.bilkent.edu.tr; gezici@ee.bilkent.edu.tr; oarikan@ ee.bilkent.edu.tr).

Color versions of one or more of the figures in this paper are available online at http://ieeexplore.ieee.org.

Digital Object Identifier 10.1109/TSP.2017.2757912 lized. A common approach for position estimation in wireless networks is to deploy a number of anchor nodes with known locations, from which target nodes estimate their locations using parameters such as time-of-arrival (TOA) and received signal strength (RSS) [5]. In order to quantify performance bounds of wireless localization systems, theoretical accuracy limits have intensely been studied in the literature; e.g., [6].

Localization accuracy of wireless networks can be reduced by employing jammer nodes over the area of interest, which aim to disrupt the position estimation process of target nodes [7]. Several studies are performed on jamming of wireless localization systems in the literature [8]-[14]. In [8], performance of GPS jamming and anti-jamming techniques are investigated, while the studies in [9], [10] focus on the design of anti-jamming algorithms for GPS receivers. In [11], optimal locations of jammers on the nodes of a uniform grid are determined in the absence of any information about the network to be jammed. Optimal attack and defense strategies from the viewpoints of the jammer and the network are analyzed for wireless sensor networks in [12], where the optimization objective is to maximize (minimize) the total delay for the jammer (network). Although jamming and anti-jamming methods and their performances have been considered in the literature, only a few studies have presented a theoretical framework to optimize the performance of jammer nodes with respect to different optimization metrics [13], [14]. In [13], two different optimization schemes with the aim of maximizing the average (max-mean scheme - Scheme 1) or the minimum (max-min scheme - Scheme 2) CRLB of target nodes are employed in order to obtain the optimal power allocation strategies for jammer nodes. The work in [14] investigates the optimal location of the jammer node that maximizes the minimum CRLB of target nodes in a non-cooperative localization network.

The work in [13] explores optimal strategies for jammer power allocation by maximizing the minimum or the average of the CRLBs of target nodes under average and peak power constraints. Since either the minimum or the average CRLB metric is considered in [13], the obtained power allocation strategy can lead to unfavorable performance in terms of the other metric not considered in the optimization. In particular, the max-min scheme adopts a conservative approach by optimizing the worst-case performance from jamming perspective (i.e., the minimum CRLB) whereas the max-mean scheme considers the average performance. However, the max-min scheme may lead to poor performance in terms of the average CRLB by considering only the worst-case performance, and the max-mean 
scheme tends to produce intolerably low CRLBs for some target nodes, which can be of critical importance from jamming perspective, by ignoring the worst-case scenario. Hence, the max-mean and max-min schemes account for only the two extreme cases of the optimal jammer power allocation problem. The motivation behind this paper is to devise a new optimization scheme which covers the max-mean and max-min schemes as special cases and balances the effects of each criterion on the overall jamming performance via a design parameter. Therefore, in this paper, a new optimization approach, namely the restricted scheme, is proposed for jammer power allocation, where the objective is to maximize the average CRLB while keeping the minimum CRLB above a predefined level. The restricted scheme is shown to be equivalent to the maximization of a linear combination of the average and the minimum CRLBs; thus it presents a trade-off between the average and worst-case performances by incorporating the minimum CRLB level into optimization constraints for average CRLB maximization. In addition, an alternative scheme is considered, where the aim is to disable as many target nodes as possible under average and peak power constraints. Even though the optimal jammer power allocation problem has been studied before in the literature, e.g., [13], novel optimization schemes are employed in this paper which provide improved jamming performance in terms of degradation of localization accuracy in a wireless localization network.

\section{B. Literature Survey on Resource Allocation}

The problem of optimal resource (e.g., power and bandwidth) allocation for wireless localization and radar systems has widely been studied in the literature [15]-[23]. In [15], the power allocation problem for anchor nodes in a wireless localization network is formulated to minimize the squared position error bound (SPEB) and the maximum directional position error bound ( $\mathrm{mD}$ PEB) regarding the position estimation of target nodes. It is shown that SPEB and mDPEB based formulations can be expressed as semidefinite programming (SDP) and second-order cone programming (SOCP) problems, respectively, which lead to fast solutions. The study in [16] investigates optimal power allocation algorithms for anchor and agent (i.e., target) nodes in a cooperative localization system by using the individual SPEB (iSPEB) as the performance metric for localization. Similarly, sensor and beacon ranging energies are minimized for a wireless sensor network in [17] via an SDP based efficient algorithm under the constraint that the CRLB for positioning does not exceed a certain threshold. In addition to power allocation schemes, several studies are carried out on joint allocation of available resources, such as power and bandwidth, in wireless localization networks [18], [19]. As a common approach, the purpose of joint resource optimization in such systems is to maximize the localization accuracy of target nodes via efficient utilization of power and spectral resources. In [18], the joint power and bandwidth allocation problem is formulated to minimize the total SPEB of agent node positioning in a cooperative localization network. Due to the nonconvex nature of the objective functions, a Taylor linearization based iterative algorithm is proposed for finding the optimal power and bandwidth distributions related to agent nodes. The study in [19] performs joint optimization of power, carrier frequency, and bandwidth allocation for agent nodes in non-cooperative wireless localization networks in order to maximize the localization accuracy by employing the single condensation (SC) method for approximating the non-convex problems as geometric programming problems.

Power allocation for radar systems, especially for distributed architectures, has drawn some attention in the literature. The authors in [21] investigate optimal power allocation strategies under total and individual power constraints in wireless sensor networks for distributed passive multiple-radar architectures. In [23], a cognitive radar network consisting of several radars used for multiple-target tracking is considered, where algorithms for optimal power allocation among multiple antennas are developed by using the posterior CRLB on target and channel state estimates as an optimization criterion. The study in [22] exploits constraint and objective relaxation of the formulated optimization problem and domain decomposition methods to determine the optimal power allocation among radar transmit powers in a distributed multiple-radar system, where both CRLB of target localization and total transmit power budget are used as performance metrics.

\section{Contributions}

In this paper, a new scheme (named as the restricted scheme) is proposed for optimal jammer power allocation in wireless localization systems. The proposed scheme is essentially based on the notion of the restricted Bayesian approach [24], [25], which covers the Bayesian and minimax approaches as special cases. The objective in the restricted Bayesian problem is to minimize the Bayes risk (average risk) under the constraint that the minimum risk is not allowed to exceed a predefined level which is specified according to the uncertainty degree in the prior probabilities (related to unknown parameters or hypotheses). In this paper, we basically build up a framework for power allocation of jammer nodes in wireless localization systems by using the notion of the restricted Bayesian approach. Even though the restricted scheme and the restricted Bayesian approach are similar from the technical point of view, the motivations, and the parameter and function definitions are completely different. In summary, by utilizing the notion of the restricted Bayesian approach, we propose a generalized power allocation criterion (named as the restricted scheme) for jammer nodes in wireless localization systems, which is a generalized criterion in the sense that it covers the criteria proposed in [13] as special cases and establishes a trade-off ground between them. The main contributions of this paper can be summarized as follows:

- The restricted scheme is proposed as a generalized optimization framework for power allocation among jammer nodes in wireless localization systems. The proposed scheme is formulated as a linear programming problem and shown to represent a trade-off between the average and the minimum CRLB optimization schemes.

- It is demonstrated that the average CRLB corresponding to the optimal solution of the restricted scheme is a strictly 
decreasing and concave function of the constraint on the minimum CRLB level.

- A closed-form power allocation solution for the restricted scheme is obtained under certain conditions on the total power limit and the design parameter that signifies the level of trade-off.

- Based on the minimax theorem, it is shown that the optimal solution of the restricted scheme contains at most $N_{T}$ jammer nodes, where $N_{T}$ is the number of target nodes, and that the optimal solution of the minimum CRLB maximization scheme is determined by at most $N_{J}$ target nodes, where $N_{J}$ is the number of jammer nodes.

- By utilizing the framework in [25], an extension to the restricted scheme is proposed to cover more generic scenarios and the corresponding optimal solution is characterized. In the extended scheme, the target nodes are grouped into subsets based on their significance levels and the constraint on the minimum CRLB level is different for each subset.

- An alternative optimization scheme is proposed in order to maximize the number of disabled (deactivated) target nodes in a given wireless localization system, where deactivation of a target node is determined according to its CRLB for localization. The solution of the alternative scheme is proved to be the same as that of the minimum CRLB maximization scheme for a specific subnetwork of target nodes.

\section{SYSTEM MODEL}

Consider a two-dimensional wireless localization system consisting of $N_{A}$ anchor nodes and $N_{T}$ target nodes located at $\boldsymbol{y}_{j} \in$ $\mathbb{R}^{2}, j=1, \ldots, N_{A}$ and $\boldsymbol{x}_{i} \in \mathbb{R}^{2}, i=1, \ldots, N_{T}$, respectively. ${ }^{1}$ In the localization scenario, self-positioning is considered [5]; that is, the target nodes are assumed to estimate their locations based on signals received from the anchor nodes with known locations. In addition to the target and anchor nodes, there exist $N_{J}$ jammer nodes at $\boldsymbol{z}_{\ell} \in \mathbb{R}^{2}, \ell=1, \ldots, N_{J}$ in the system, which aim to degrade the localization performance of the system. In compliance with the common approach in the literature [26]-[28], the jammer nodes are assumed to transmit zero-mean Gaussian noise.

In this paper, there exists no cooperation among the target nodes; that is, the target nodes receive signals only from the anchor nodes (i.e., not from the other target nodes) for position estimation. In addition, the connectivity sets are defined as $\mathcal{A}_{i} \triangleq$ $\left\{j \in\left\{1, \ldots, N_{A}\right\} \mid\right.$ anchor node $j$ is connected to target node $\left.i\right\}$ for $i \in\left\{1, \ldots, N_{T}\right\} .^{2}$ Then, the received signal at target node $i$ coming from anchor node $j$ can be expressed as [13]

$$
r_{i j}(t)=\sum_{k=1}^{L_{i j}} \alpha_{i j}^{k} s_{j}\left(t-\tau_{i j}^{k}\right)+\sum_{\ell=1}^{N_{J}} \gamma_{i \ell} \sqrt{P_{\ell}^{J}} v_{i j \ell}(t)+n_{i j}(t)
$$

\footnotetext{
${ }^{1}$ Generalizations to the three-dimensional scenario are straightforward, but not explored in this study.

${ }^{2} \mathrm{An}$ anchor node being connected to a target node means that the receiver of a target node will be able to decode the signal coming from an anchor node, which happens only when the SNR is above a threshold.
}

for $t \in\left[0, T_{\text {obs }}\right], i \in\left\{1, \ldots, N_{T}\right\}$, and $j \in \mathcal{A}_{i}$, where $T_{\text {obs }}$ is the observation time, $\alpha_{i j}^{k}$ and $\tau_{i j}^{k}$ denote, respectively, the amplitude and delay of the $k$ th multipath component between anchor node $j$ and target node $i, L_{i j}$ is the number of paths between target node $i$ and anchor node $j$, and $\gamma_{i \ell}$ represents the channel coefficient between target node $i$ and jammer node $\ell$, which has a transmit power of $P_{\ell}^{J}$. ${ }^{3}$ The transmit signals $s_{j}(t)$ 's are assumed to be known and orthogonal, and the measurement noise $n_{i j}(t)$ and the jammer noise $\sqrt{P_{\ell}^{J}} v_{i j \ell}(t)$ are assumed to be independent zero-mean white Gaussian random processes, where the average power of $n_{i j}(t)$ is $N_{0} / 2$ and that of $v_{i j \ell}(t)$ is equal to one. In addition, for each target node $i, n_{i j}(t)$ is independent for $j \in \mathcal{A}_{i}$, and $v_{i j \ell}(t)$ is independent for $\ell \in\left\{1,2, \ldots, N_{J}\right\}$ and for $j \in \mathcal{A}_{i} \cdot{ }^{4}$ The delay term $\tau_{i j}^{k}$ in (1) can be expressed as

$$
\tau_{i j}^{k} \triangleq \frac{\left\|\boldsymbol{y}_{j}-\boldsymbol{x}_{i}\right\|+b_{i j}^{k}}{c}
$$

where $b_{i j}^{k} \geq 0$ and $c$ denote, respectively, the range bias and the speed of propagation. For the paths between anchor node $j$ and target node $i$, the range bias $b_{i j}^{k}$ describes the difference between the actual travel distance of the $k$ th signal path and the distance between nodes. Set $\mathcal{A}_{i}$ is partitioned as

$$
\mathcal{A}_{i} \triangleq \mathcal{A}_{i}^{L} \cup \mathcal{A}_{i}^{N L}
$$

where $\mathcal{A}_{i}^{L}$ and $\mathcal{A}_{i}^{N L}$ represent the sets of anchors nodes with line-of-sight (LOS) and non-line-of-sight (NLOS) connections to target node $i$, respectively.

In order to derive the CRLB for localization of the target nodes, the unknown parameter vector for target node $i$ must be specified. The vector consisting of the bias terms related to target node $i$ in the LOS and NLOS cases is given by

$$
\boldsymbol{b}_{i j}=\left\{\begin{array}{ll}
{\left[b_{i j}^{2} \ldots b_{i j}^{L_{i j}}\right]^{T},} & \text { if } j \in \mathcal{A}_{i}^{L} \\
{\left[b_{i j}^{1} \ldots b_{i j}^{L_{i j}}\right]^{T},} & \text { if } j \in \mathcal{A}_{i}^{N L}
\end{array} .\right.
$$

Based on (4), the unknown parameters related to target node $i$ are defined as [29]

$$
\boldsymbol{\theta}_{i} \triangleq\left[\boldsymbol{x}_{i}^{T} \boldsymbol{b}_{i \mathcal{A}_{i}(1)}^{T} \cdots \boldsymbol{b}_{i \mathcal{A}_{i}\left(\left|\mathcal{A}_{i}\right|\right)}^{T} \boldsymbol{\alpha}_{i \mathcal{A}_{i}(1)}^{T} \cdots \boldsymbol{\alpha}_{i \mathcal{A}_{i}\left(\left|\mathcal{A}_{i}\right|\right)}^{T}\right]^{T}
$$

where $\mathcal{A}_{i}(j)$ denotes the $j$ th element of set $\mathcal{A}_{i},\left|\mathcal{A}_{i}\right|$ represents the number of elements in $\mathcal{A}_{i}$, and $\boldsymbol{\alpha}_{i j} \triangleq\left[\alpha_{i j}^{1} \cdots \alpha_{i j}^{L_{i j}}\right]^{T}$. It is assumed that the total noise level is known by each target node [14]..$^{5}$

\footnotetext{
${ }^{3}$ The channel coefficient $\gamma_{i \ell}$ between target node $i$ and jammer node $\ell$ is modeled to be independent of the anchor node index based on the assumptions that time division multiple access is employed for communications of different anchor nodes with target node $i$ and the channel coefficient $\gamma_{i \ell}$ does not change considerably over the time slots.

${ }^{4}$ Multiple access techniques make $v_{i j \ell}(t)$ 's independent over the anchor nodes; i.e., for $j \in \mathcal{A}_{i}$ (Remark 1 in [14]).

${ }^{5}$ This assumption enables target nodes to employ the maximum likelihood (ML) estimator for localization, which involves the total noise levels corresponding to different anchor nodes. Since the ML estimator asymptotically converges to the CRLB as the SNR and/or effective bandwidth increases [30], it is reasonable to assume that target nodes perform position estimation using an ML estimator. In that case, the CRLB as a performance metric represents a localization accuracy that is close to the accuracy attained by target nodes (see Remark 3 for a detailed discussion).
} 
The CRLB for location estimation of target node $i$ is provided by [4]

$$
\mathbb{E}\left\{\left\|\hat{\boldsymbol{x}}_{i}-\boldsymbol{x}_{i}\right\|^{2}\right\} \geq \operatorname{tr}\left\{\left[\boldsymbol{F}_{i}^{-1}\right]_{2 \times 2}\right\}
$$

where $\hat{\boldsymbol{x}}_{i}$ denotes an unbiased estimate of the location of target node $i, \operatorname{tr}\{\cdot\}$ represents the trace operator, and $\boldsymbol{F}_{i}$ is the Fisher information matrix for vector $\boldsymbol{\theta}_{i}$. Following the derivations performed in [6], $\left[\boldsymbol{F}_{i}^{-1}\right]_{2 \times 2}$ can be expressed as

$$
\left[\boldsymbol{F}_{i}^{-1}\right]_{2 \times 2}=\boldsymbol{J}_{i}\left(\boldsymbol{x}_{i}, \boldsymbol{p}^{J}\right)^{-1}
$$

where the equivalent Fisher information matrix $\boldsymbol{J}_{i}\left(\boldsymbol{x}_{i}, \boldsymbol{p}^{J}\right)$ in the absence of prior information about the location of the target node is calculated as

$$
\boldsymbol{J}_{i}\left(\boldsymbol{x}_{i}, \boldsymbol{p}^{J}\right)=\sum_{j \in \mathcal{A}_{i}^{L}} \frac{\lambda_{i j}}{N_{0} / 2+\boldsymbol{a}_{i}^{T} \boldsymbol{p}^{J}} \boldsymbol{\phi}_{i j} \boldsymbol{\phi}_{i j}^{T}
$$

with

$$
\begin{aligned}
\lambda_{i j} & \triangleq \frac{4 \pi^{2} \beta_{j}^{2}\left|\alpha_{i j}^{1}\right|^{2} \int_{-\infty}^{\infty}\left|S_{j}(f)\right|^{2} d f}{c^{2}}\left(1-\xi_{i j}\right), \\
\boldsymbol{a}_{i} & \triangleq\left[\left|\gamma_{i 1}\right|^{2} \cdots\left|\gamma_{i N_{J}}\right|^{2}\right]^{T}, \\
\boldsymbol{p}^{J} & \triangleq\left[P_{1}^{J} \cdots P_{N_{J}}^{J}\right]^{T}, \\
\boldsymbol{\phi}_{i j} & \triangleq\left[\begin{array}{ll}
\cos \varphi_{i j} & \sin \varphi_{i j}
\end{array}\right]^{T} .
\end{aligned}
$$

In (9), $\beta_{j}$ is the effective bandwidth, which is expressed as $\beta_{j}=$ $\sqrt{\int_{-\infty}^{\infty} f^{2}\left|S_{j}(f)\right|^{2} d f / \int_{-\infty}^{\infty}\left|S_{j}(f)\right|^{2} d f}$, with $S_{j}(f)$ denoting the Fourier transform of $s_{j}(t)$, and the path-overlap coefficient $\xi_{i j}$ is a non-negative number between zero and one, i.e., $0 \leq \xi_{i j} \leq 1$ [6]. Also, in (12), $\varphi_{i j}$ denotes the angle between target node $i$ and anchor node $j$. In addition, it is assumed that the elements of $\boldsymbol{a}_{i}$ are non-zero (i.e., strictly positive) for $i \in\left\{1,2, \ldots, N_{T}\right\}$. It is noted from (8) that the effects of the jammer nodes appear as the second term in the denominator since the jammer nodes transmit Gaussian noise.

According to Lemma 1 in [13], the trace of the inverse of the equivalent Fisher information matrix $\boldsymbol{J}_{i}\left(\boldsymbol{x}_{i}, \boldsymbol{p}^{\boldsymbol{J}}\right)$ in (8); equivalently, the CRLB for target node $i$, is an affine function with respect to $\boldsymbol{p}^{J}$, which is expressed as

$$
\operatorname{tr}\left\{\boldsymbol{J}_{i}\left(\boldsymbol{x}_{i}, \boldsymbol{p}^{J}\right)^{-1}\right\}=r_{i} \boldsymbol{a}_{i}^{T} \boldsymbol{p}^{J}+r_{i} N_{0} / 2 \triangleq C_{i}\left(\boldsymbol{p}^{J}\right)
$$

where

$$
r_{i} \triangleq \operatorname{tr}\left\{\left[\sum_{j \in \mathcal{A}_{i}^{L}} \lambda_{i j} \boldsymbol{\phi}_{i j} \boldsymbol{\phi}_{i j}^{T}\right]^{-1}\right\} .
$$

Remark 1: The dependency of the Fisher information matrix on network geometry (i.e., locations of anchor, target and jammer nodes) and jammer powers can be scrutinized by considering the expression in (8) as follows: The Fisher information matrix in (8) for the location of target node $i$ depends on the target location $\boldsymbol{x}_{i}$ via the parameters $\lambda_{i j}, \boldsymbol{a}_{i}$, and $\phi_{i j}$, and on the jammer powers via the power vector $\boldsymbol{p}^{J}$. First, from (9), the amplitude of the LOS path $\alpha_{i j}^{1}$ and the path-overlap coefficient $\xi_{i j}$ between target node $i$ and anchor node $j$ depend on $\boldsymbol{x}_{i}$ and $\boldsymbol{y}_{j}$. Next, $\boldsymbol{a}_{i}$ in (10) consists of the channel gains between target node $i$ and jammer nodes, which depend on $\boldsymbol{x}_{i}$ and $\boldsymbol{z}_{\ell}$ for $\ell \in\left\{1, \ldots, N_{J}\right\}$. In addition, the parameter $\phi_{i j}$ in (12) is determined by the angles $\varphi_{i j}$, which are also location dependent. Therefore, both network geometry and jammer powers affect the performance of jamming strategies.

Remark 2: The role played by multipath propagation in a practical localization scenario can be explained as follows: The parameters affected by the network configuration (i.e., anchor, target and jammer positions) in the CRLB expression for target node $i$ in (8) are $\boldsymbol{a}_{i}, \boldsymbol{\phi}_{i j}$, and $\lambda_{i j}$. As discussed in Remark 1, $\boldsymbol{a}_{i}$ contains the channel gains between target node $i$ and jammer nodes and thus has no relevance to the multipath scenario. Hence, varying jammer positions does not alter the multipath conditions between the anchor and target nodes. Secondly, $\phi_{i j}$ depends on the angle $\varphi_{i j}$ between target node $i$ and anchor node $j$ via (12). Since $\varphi_{i j}$ is determined by the network geometry, changing the value of $\varphi_{i j}$, imposed by a change in the network geometry, will affect multipath conditions (i.e., delays and amplitudes of multipath components). In addition, the parameter $\lambda_{i j}$ in (9) also reflects the multipath effect via the path-overlap coefficients $\xi_{i j}$, which are determined by the transmitted waveforms $s_{j}(t)$ and the delays $\tau_{i j}^{k}$ for $j \in \mathcal{A}_{i}^{L}$ [6, Appendix III-A]. According to [6, Corollary 1], $\xi_{i j}=0$ is obtained if the first path is resolvable, i.e., the delay between the LOS path and the first arriving NLOS path exceeds the duration of $s_{j}(t)$. Depending on the amount of overlap between the LOS path and the NLOS paths, $\xi_{i j}$ takes values in the interval $[0,1]$. Hence, the multipath scenario characterizes the value of $\xi_{i j}$, which, in turn, affects the CRLB via $\lambda_{i j}$. Therefore, the effect of varying anchor node positions on the CRLB can be manifested in the angles $\varphi_{i j}$ and the multipath delays $\tau_{i j}^{k}$ between target nodes and anchor nodes.

Remark 3: The CRLB is considered as the performance metric for target location estimation since it provides a tight bound on the mean-squared error (MSE) of the ML estimator for high effective bandwidths, $\beta_{j}$, and/or high SNRs [30]. Hence, the CRLB serves as a meaningful performance benchmark for highaccuracy localization scenarios. When neither the SNRs nor the effective bandwidths are sufficiently high, the performance of the ML estimator may deviate from the CRLB [31]. These conclusions are also valid in the presence of multipath since the considered CRLB expression based on (8) and (9) takes the multipath effects into account via the path-overlap coefficients, $\xi_{i j}$ in (9) [6]. In other words, when the ML estimator is designed for the multipath scenario and the CRLB is calculated via (8) and (9), the CRLB again provides a tight bound on the MSE of the ML estimator for high effective bandwidths and/or SNRs. When neither the SNRs nor the effective bandwidths are high, the CRLB is not tight in general and the gap between the CRLB and the MSE of the ML estimator depends on specific multipath conditions. In this study, the optimized CRLB constitutes a lower bound on the target localization performance of the ML estimator when the optimal jamming strategies are employed by the jammer network. Optimal jammer power allocation solutions obtained via CRLB optimization provide essential guidelines for developing efficient jamming strategies. Moreover, the choice of CRLB as an optimization metric is also motivated by 


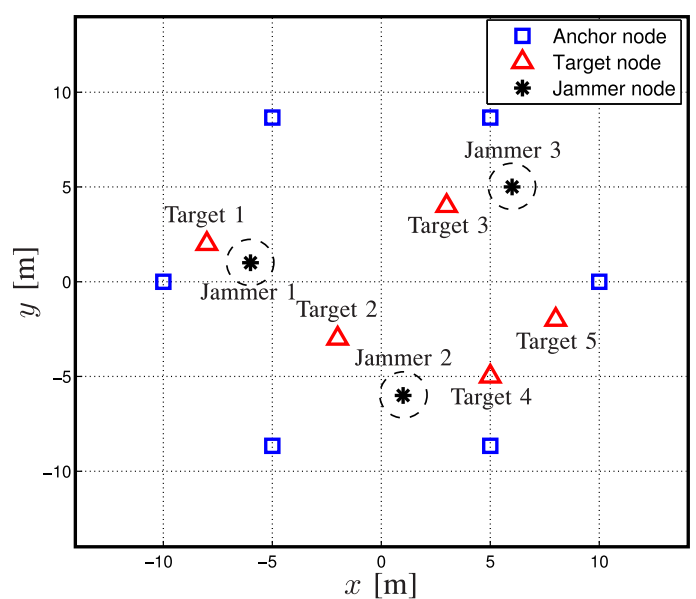

Fig. 1. The network considered in the simulations, where the anchor node positions are $[-100],[-5-5 \sqrt{3}],[-55 \sqrt{3}],[55 \sqrt{3}],[5-5 \sqrt{3}]$, and $[100] \mathrm{m}$., the target node positions are $[-82],[-2-3],[34],[5-5]$ and $[8-2] \mathrm{m}$., and the jammer node positions are $[-61],[1-6]$, and $[65] \mathrm{m}$.

its decent mathematical structure that favors theoretical characterizations (e.g., [13], [15], [17], [19], [32]).

\section{RESTRICTED SCHEME}

In this section, one of the proposed schemes, namely the restricted scheme, is introduced. The restricted scheme is an optimal power allocation strategy for jammer nodes in a wireless localization system, which covers both Scheme 1 (the maxmean scheme) and Scheme 2 (the max-min scheme), proposed in [13], as special cases. For a given wireless localization system (see, e.g., Fig. 1), Scheme 1 attempts to maximize the average CRLB of target nodes while Scheme 2 considers the minimum CRLB as the jamming performance metric. In what follows, the formulations for Scheme 1 and Scheme 2 are revisited with certain modifications, and the restricted scheme is proposed as a generalization of Scheme 1 and Scheme 2. All the schemes are formulated by imposing average (total) and peak jammer power constraints, as considered in [13]. Also, in accordance with practical systems, it is assumed that the total power limit for the jamming network is lower than the sum of the peak power limits for the jammer nodes.

Since the elements of $\boldsymbol{a}_{i}$ in (10) are positive, the CRLBs of the target nodes in (13) monotonically increase with jammer powers. Therefore, similar to Scheme 1 and Scheme 2 in [13], the restricted scheme has the full total power utilization property, meaning that its optimal solution always operates at the average (total) power limit (cf. Lemma 3 in [13]). Therefore, all the power vectors $\boldsymbol{p}^{J}$ (see (11)) in this study are assumed to satisfy $\mathbf{1}^{T} \boldsymbol{p}^{J}=P_{T}$, where $P_{T}$ represents the total power limit. Hence, in the following optimization problems, the total (equivalently, the average) power constraint is represented by $\mathbf{1}^{T} \boldsymbol{p}^{J}=P_{T}$ instead of $\mathbf{1}^{T} \boldsymbol{p}^{J} \leq P_{T}$. Also, due to the full total power utilization property, the CRLB for target node $i$ can be expressed via (13) as

$$
C_{i}\left(\boldsymbol{p}^{J}\right)=\boldsymbol{d}_{i}^{T} \boldsymbol{p}^{J}
$$

where

$$
\boldsymbol{d}_{i} \triangleq r_{i} \boldsymbol{a}_{i}+r_{i} \frac{N_{0}}{2 P_{T}} \mathbf{1} .
$$

Let $g[i]$ denote a probability mass function (PMF) defined over the set $\left\{1,2, \ldots, N_{T}\right\}$, which describes the significance level of each target node. Then, the average CRLB is calculated as follows:

$$
C_{\text {avg }}\left(\boldsymbol{p}^{J}\right)=\sum_{i=1}^{N_{T}} g[i] C_{i}\left(\boldsymbol{p}^{J}\right) .
$$

In Scheme 1 in [13], all the target nodes are assumed to have the same significance level in terms of jamming performance and are thus considered to contribute equally to the average (overall) CRLB. However, in some cases, jamming some target nodes may have priority over jamming others. Therefore, it is important to calculate the average CRLB by taking the significance level of each target node into account. The significance level of a target node can be specified by the characteristics of a target or by the region occupied by it (i.e., some regions may be more critical than others from the jamming standpoint). The expression in (17) generalizes the average CRLB formulation in [13], which considers only the uniform PMF, to an arbitrary PMF $g[i]$. Accordingly, Scheme 1 in [13] is reformulated as follows (cf. [13, Eq. (16)]):

$$
\begin{aligned}
& \underset{\boldsymbol{p}^{J}}{\operatorname{maximize}} C_{\mathrm{avg}}\left(\boldsymbol{p}^{J}\right) \\
& \text { subject to } \mathbf{1}^{T} \boldsymbol{p}^{J}=P_{T} \\
& \qquad 0 \leq P_{\ell}^{J} \leq P_{\ell}^{\text {peak }}, \ell=1,2, \ldots, N_{J} .
\end{aligned}
$$

The solution of (18) is given by that of the original version of Scheme 1 in Proposition 2 of [13] with $\boldsymbol{w}$ being redefined as $\boldsymbol{w} \triangleq \sum_{i=1}^{N_{T}} g[i] \boldsymbol{d}_{i}$. Namely, the solution assigns all the available power to the jammer node corresponding to the first, second, third, ...largest element of $\boldsymbol{w}$ under the peak power limit and until the average (total) power limit is reached (see Eq. (18) in [13]).

Before formulating Scheme 2 for power allocation among jammer nodes, the definition of the least-favorable PMF is provided as follows:

Definition 3.1: Let $g[i]$ be a PMF over the set $\left\{1,2, \ldots, N_{T}\right\}$ and $\boldsymbol{p}_{*}^{J}$ denote the corresponding optimal solution of (18). Then, $g[i]$ is defined as the least-favorable PMF if it satisfies

$$
\sum_{i=1}^{N_{T}} g[i] C_{i}\left(\boldsymbol{p}_{*}^{J}\right)=\min _{i \in\left\{1,2, \ldots, N_{T}\right\}} C_{i}\left(\boldsymbol{p}_{*}^{J}\right) .
$$

Remark 4: The least-favorable PMF $g[i]$ minimizes the average CRLB among all PMFs defined over the set $\left\{1,2, \ldots, N_{T}\right\}$ (see Appendix A for the proof).

Scheme 2 considers the minimum CRLB as a performance metric, which is in fact the average CRLB calculated based on the least-favorable significance level distribution (from the jamming standpoint) over the target nodes (i.e, the least-favorable $g[i]$ according to Definition 3.1). Scheme 2 is regarded as a 
conservative approach since it considers the worst-case scenario (in terms of jamming performance), which occurs when $g[i]$ corresponds to the least-favorable PMF. The formulation of Scheme 2 is provided as follows [13, Eq. (19)]:

$$
\begin{aligned}
& \underset{\boldsymbol{p}^{J}}{\operatorname{maximize}} \min _{i \in\left\{1,2, \ldots, N_{T}\right\}} C_{i}\left(\boldsymbol{p}^{J}\right) \\
& \text { subject to } \mathbf{1}^{T} \boldsymbol{p}^{J}=P_{T} \\
& 0 \leq P_{\ell}^{J} \leq P_{\ell}^{\text {peak }}, \ell=1,2, \ldots, N_{J} .
\end{aligned}
$$

Remark 5: It is noted from Definition 3.1 that Scheme 1 and Scheme 2 become equivalent for the least-favorable PMF (see Appendix B for the proof).

It should be emphasized that $g[i]$ represents the relative significance level of each target node with respect to the other target nodes; hence, the average CRLB is mainly dependent on the PMF $g[i]$. On the other hand, the minimum CRLB is an absolute metric in the sense that it is not affected by the relative relations among the target nodes (that is, it is only dependent on the worst-case scenario). Therefore, it can be concluded that the average and minimum CRLBs are not alternatives to each other; that is, they represent two completely different facets of a design problem. In general, even if $g[i]$ is not the same as the least-favorable PMF, the minimum CRLB being lower than some level can be intolerable due to design requirements. To that end, a new scheme, namely the restricted scheme, is proposed for the aim of maximizing the average CRLB while keeping the minimum CRLB above a preset level. The restricted scheme is formulated as follows: ${ }^{6}$

$$
\begin{array}{ll}
\underset{\boldsymbol{p}^{J}}{\operatorname{maximize}} & C_{\mathrm{avg}}\left(\boldsymbol{p}^{J}\right) \\
\text { subject to } & \min _{i \in\left\{1,2, \ldots, N_{T}\right\}} C_{i}\left(\boldsymbol{p}^{J}\right) \geq \alpha \\
& \mathbf{1}^{T} \boldsymbol{p}^{J}=P_{T} \\
& 0 \leq P_{\ell}^{J} \leq P_{\ell}^{\text {peak }}, \ell=1,2, \ldots, N_{J}
\end{array}
$$

where $\alpha$ is a design parameter preset according to design requirements. In the restricted scheme, both the average and the worst-case performances are taken into account, and the design parameter can be adjusted to determine the effect of each performance metric on the overall jamming performance. It should be noted that (21) covers both (18) and (20) as special cases: The restricted scheme reduces to Scheme 2 if $\alpha$ takes its maximum value $\bar{\alpha}$ which corresponds to the solution of (20) (i.e., $\bar{\alpha} \triangleq \min _{i \in\left\{1,2, \ldots, N_{T}\right\}} C_{i}\left(\overline{\boldsymbol{p}}^{J}\right)$, where $\overline{\boldsymbol{p}}^{J}$ is the solution of (20)), and reduces to Scheme 1 if the value of $\alpha$ is lower than or equal to the value $\underline{\alpha}$ corresponding to the solution of (18) (i.e., $\underline{\alpha} \triangleq$ $\min _{i \in\left\{1,2, \ldots, N_{T}\right\}} C_{i}\left(\underline{\boldsymbol{p}}^{J}\right)$, where $\underline{\boldsymbol{p}}^{J}$ is the solution of (18)). The formulation in (21) can be expressed as a linear programming (LP) problem as is the case for both Scheme 1 and Scheme 2.

\footnotetext{
${ }^{6}$ This formulation is similar to that of the restricted Bayes [24], [25], which includes both Bayesian and minimax problems as special cases.
}

Equivalently, the restricted scheme can also be formulated as follows:

$$
\begin{aligned}
& \underset{\boldsymbol{p}^{J}}{\operatorname{maximize}} \lambda C_{\mathrm{avg}}\left(\boldsymbol{p}^{J}\right)+(1-\lambda) \min _{i} C_{i}\left(\boldsymbol{p}^{J}\right) \\
& \text { subject to } \mathbf{1}^{T} \boldsymbol{p}^{J}=P_{T} \\
& \qquad 0 \leq P_{\ell}^{J} \leq P_{\ell}^{\text {peak }}, \ell=1,2, \ldots, N_{J}
\end{aligned}
$$

where $0 \leq \lambda \leq 1$, similar to $\alpha$, is specified according to design requirements $[25]^{7}$

\section{PROPERTIES OF OPtiMAl SOLUTION FOR RESTRICTED SCHEME}

In this section, the aim is to characterize the optimal solution for the restricted scheme formulations in (21) and (22). The following proposition investigates the characteristics of the optimal solution of (22) and establishes a link between the optimal solutions of (18) and (22).

Proposition 4.1: Define a PMF $v[i]$ as $v[i] \triangleq \lambda g[i]+(1-$ $\lambda$ ) $f[i]$, where $f[i]$ is any valid PMF defined over the set $\left\{1,2, \ldots, N_{T}\right\}$. If $\boldsymbol{p}_{*}^{J}$ is the solution of Scheme 1 for $v[i]$ and satisfies

$$
\sum_{i=1}^{N_{T}} f[i] C_{i}\left(\boldsymbol{p}_{*}^{J}\right)=\min _{i \in\left\{1,2, \ldots, N_{T}\right\}} C_{i}\left(\boldsymbol{p}_{*}^{J}\right),
$$

then it is a solution of (22) for $g[i]$.

Proof: The steps in this proof are similar to those in $[25$, Theorem 1]. Let $\boldsymbol{p}^{J}$ represent any power vector satisfying the total and peak power constraints. Then,

$$
\begin{aligned}
& \lambda C_{\mathrm{avg}}\left(\boldsymbol{p}^{J}\right)+(1-\lambda) \min _{i \in\left\{1,2, \ldots, N_{T}\right\}} C_{i}\left(\boldsymbol{p}^{J}\right) \\
\leq & \lambda \sum_{i=1}^{N_{T}} g[i] C_{i}\left(\boldsymbol{p}^{J}\right)+(1-\lambda) \sum_{i=1}^{N_{T}} f[i] C_{i}\left(\boldsymbol{p}^{J}\right) \\
= & \sum_{i=1}^{N_{T}}(\lambda g[i]+(1-\lambda) f[i]) C_{i}\left(\boldsymbol{p}^{J}\right) \\
\leq & \sum_{i=1}^{N_{T}}(\lambda g[i]+(1-\lambda) f[i]) C_{i}\left(\boldsymbol{p}_{*}^{J}\right) \\
= & \lambda C_{\mathrm{avg}}\left(\boldsymbol{p}_{*}^{J}\right)+(1-\lambda) \underset{i \in\left\{1,2, \ldots, N_{T}\right\}}{\min } C_{i}\left(\boldsymbol{p}_{*}^{J}\right)
\end{aligned}
$$

where the first inequality due to the relation between the minimum and the average operations, $\boldsymbol{p}_{*}^{J}$ (the solution of Scheme 1 for $v[i]$ ) is employed to proceed from (26) to (27), and the condition (23) in the proposition is used to obtain (28) from (27). Overall, the relation between (24) and (28) indicate that $\boldsymbol{p}_{*}^{J}$ is a solution of (22) for $g[i]$.

The optimal solution specified by Proposition 4.1 always exists since the probability distributions are discrete and defined over a compact set [25], [33]. It can be noted based on

\footnotetext{
${ }^{7}$ The proof of equivalence of the optimization schemes in (21) and (22) will be provided in Section IV.
} 
Proposition 4.1 that the solution of (22) can be obtained by finding a PMF $f[i]$ for which (23) is satisfied for the power vector corresponding to Scheme 1 for $v[i]$, which has a closed-form solution [13]. Hence, the solution of the restricted scheme formulation in (22) reduces to determining a PMF $f[i]$ such that the conditions in Proposition 4.1 are satisfied. This proposition also emphasizes the equalizer nature of the restricted scheme. For example, if $f[i]$ consists of three point masses at $i=1,3$, and 4 , it implies based on the proposition that the CRLBs of target nodes 1,3 , and 4 are equalized to the minimum CRLB of the whole network.

The following corollary shows that the formulations in (21) and (22) of the restricted scheme are equivalent to each other, and forms a formal link between them.

Corollary 4.2: Under the conditions in Proposition 4.1, $\boldsymbol{p}_{*}^{J}$ solves the optimization problem in (21) when the design parameter satisfies $\alpha=\min _{i \in\left\{1,2, \ldots, N_{T}\right\}} C_{i}\left(\boldsymbol{p}_{*}^{J}\right)$.

Proof: The proof follows similar steps to those in [25, Corollary]. For any $\boldsymbol{p}^{J}$ satisfying the constraints in (21), Proposition 4.1 implies the following inequality:

$$
\begin{aligned}
& \lambda C_{\mathrm{avg}}\left(\boldsymbol{p}^{J}\right)+(1-\lambda) \min _{i \in\left\{1,2, \ldots, N_{T}\right\}} C_{i}\left(\boldsymbol{p}^{J}\right) \\
\leq & \lambda C_{\mathrm{avg}}\left(\boldsymbol{p}_{*}^{J}\right)+(1-\lambda) \min _{i \in\left\{1,2, \ldots, N_{T}\right\}} C_{i}\left(\boldsymbol{p}_{*}^{J}\right) .
\end{aligned}
$$

It is given that $\min _{i \in\left\{1,2, \ldots, N_{T}\right\}} C_{i}\left(\boldsymbol{p}_{*}^{J}\right)=\alpha$, thus the inequality $\min _{i \in\left\{1,2, \ldots, N_{T}\right\}} C_{i}\left(\boldsymbol{p}^{J}\right) \geq \alpha$ with (29) results in $C_{\text {avg }}\left(\boldsymbol{p}_{*}^{J}\right) \geq$ $C_{\text {avg }}\left(\boldsymbol{p}^{j}\right)$.

Corollary 4.2 establishes a formal link between parameters $\lambda$ and $\alpha$; that is, for any $\lambda, \alpha$ can be calculated through the equation in the corollary. More specifically, the optimization problem in (22) is solved for a given $\lambda$ and the optimal solution $p^{J}(\lambda)$ is inserted into the equation $\min _{i \in\left\{1,2, \ldots, N_{T}\right\}} C_{i}\left(\boldsymbol{p}^{J}(\lambda)\right)=\alpha$ to obtain $\alpha$ for a given $\lambda$.

In the following proposition, it is shown that the optimal solution of the restricted scheme is the solution of Scheme 1 for the least-favorable PMF $v[i]$ among a family of PMFs [25, Theorem 2]. This result reveals an important property of the PMF $v[i]$ as defined in Proposition 4.1, for which Scheme 1 is to be solved in order to obtain the optimal solution of the restricted scheme.

Proposition 4.3: Under the conditions in Proposition 4.1, $v[i]=\lambda g[i]+(1-\lambda) f[i]$ minimizes the average CRLB among all PMFs in the form of $\tilde{v}[i]=\tilde{\lambda} g[i]+(1-\tilde{\lambda}) \tilde{f}[i]$ for $\tilde{\lambda} \geq \lambda$, where $\tilde{f}[i]$ is any valid PMF defined over the set $\left\{1,2, \ldots, N_{T}\right\}$. Equivalently,

$$
\sum_{i=1}^{N_{T}} v[i] C_{i}\left(\boldsymbol{p}_{*}^{J}\right) \leq \sum_{i=1}^{N_{T}} \tilde{v}[i] C_{i}\left(\boldsymbol{p}_{+}^{J}\right)
$$

for any $\tilde{v}[i]$ described above, where $\boldsymbol{p}_{*}^{J}$ and $\boldsymbol{p}_{+}^{J}$ are the solutions of Scheme 1 for $v[i]$ and $\tilde{v}[i]$, respectively.

Proof: See Appendix C.

It is noted that when $\lambda=0$, the restricted scheme in (22) is equivalent to Scheme 2 and the family of PMFs among which $f[i]$ minimizes the average CRLB becomes the set of all PMFs defined over the set $\left\{1,2, \ldots, N_{T}\right\}(\tilde{v}[i]$ represents the set of all valid PMFs by taking $\tilde{\lambda}=0$ ). Therefore, as a special case of Proposition 4.3, it can be deduced that Scheme 2 is equivalent to Scheme 1 if $g[i]$ is the least-favorable PMF among all PMFs defined over the set $\left\{1,2, \ldots, N_{T}\right\}$, which is in compliance with Remark 5.

Next, the characteristics of the average CRLB of the restricted scheme is investigated with respect to the changes in $\alpha$. Let $\boldsymbol{p}_{r}^{J}, \boldsymbol{p}_{1}^{J}$, and $\boldsymbol{p}_{2}^{J}$ denote the solutions of the restricted scheme, Scheme 1, and Scheme 2, respectively. Also, let $\underline{\alpha} \triangleq$ $\min _{i \in\left\{1,2, \ldots, N_{T}\right\}} C_{i}\left(\boldsymbol{p}_{1}^{J}\right)$ and $\bar{\alpha} \triangleq \min _{i \in\left\{1,2, \ldots, N_{T}\right\}} C_{i}\left(\boldsymbol{p}_{2}^{J}\right)$ define the minimum CRLBs of Scheme 1 and Scheme 2, respectively. In particular, $\bar{\alpha}$ is the maximum value that $\alpha$ can take due to the definition of Scheme 2; that is, when $\alpha=\bar{\alpha}$, the restricted scheme reduces to Scheme 2. For $0 \leq \alpha \leq \underline{\alpha}$, the constraint on the minimum CRLB in (21) becomes ineffective, which results in that the restricted scheme reduces to Scheme 1 for $0 \leq \alpha \leq \underline{\alpha}$. Accordingly, $C_{\mathrm{avg}}\left(\boldsymbol{p}_{r}^{J}\right)$ is constant and equal to $C_{\text {avg }}\left(\boldsymbol{p}_{1}^{J}\right)$ for $0 \leq \alpha \leq \underline{\alpha}$. Therefore, in practice, $\alpha$ is an element of the closed interval $[\underline{\alpha}, \bar{\alpha}]$; that is, $\alpha \in[\underline{\alpha}, \bar{\alpha}]$. The following proposition characterizes the behavior of the average CRLB of the restricted scheme with respect to $\alpha \in[\underline{\alpha}, \bar{\alpha}]$.

Proposition 4.4: The average CRLB of the restricted scheme, $C_{\text {avg }}\left(\boldsymbol{p}_{r}^{J}(\alpha)\right)$, is a strictly decreasing and concave function of $\alpha$ for $\alpha \in[\underline{\alpha}, \bar{\alpha}]$.

Proof: See Appendix D.

The following corollary, which is obtained based on Proposition 4 in [13], Proposition 4.1, and Corollary 4.2, presents a closed-form solution for the restricted scheme under certain conditions on $\lambda$ and $P_{T}$.

Corollary 4.5: Let $\zeta_{n}$ for $n \in\left\{1,2, \ldots, N_{J}\right\}$ be defined as follows:

$$
\zeta_{n} \triangleq \frac{r_{k}\left(\left|\gamma_{k b}\right|^{2}-\left|\gamma_{k n}\right|^{2}\right)}{\sum_{i=1}^{N_{T}} g[i] r_{i}\left(\left|\gamma_{i n}\right|^{2}-\left|\gamma_{i b}\right|^{2}\right)+r_{k}\left(\left|\gamma_{k b}\right|^{2}-\left|\gamma_{k n}\right|^{2}\right)}
$$

where $k$ is the target node that uniquely has the minimum CRLB in the absence of jamming and $b$ is given by

$$
b=\arg \max _{\ell \in\left\{1,2, \ldots, N_{J}\right\}}\left|\gamma_{k \ell}\right|^{2} .
$$

Also, define $\tilde{\zeta}$ as

$$
\tilde{\zeta}=\min _{n \in \mathcal{J}} \zeta_{n}
$$

where the set $\mathcal{J}$ is given by

$$
\mathcal{J}=\left\{n \in\left\{1,2, \ldots, N_{J}\right\} \mid n \neq b \text { and } \zeta_{n} \geq 0\right\} .
$$

Then, $0 \leq \lambda \leq \tilde{\zeta}$ corresponds to $\alpha=\bar{\alpha}$ if the total power limit satisfies $P_{T} \leq P_{T}^{(k)}$, where $P_{T}^{(k)}$ is described in [13, Eq. 22] (assuming that $P_{b}^{\text {peak }} \geq P_{T}$ ). In other words, when $0 \leq \lambda \leq$ $\tilde{\zeta}$, the restricted scheme and Scheme 2 become identical for $P_{T} \leq P_{T}^{(k)}$. In addition, the solution of both schemes under the conditions $0 \leq \lambda \leq \tilde{\zeta}$ and $P_{T} \leq P_{T}^{(k)}$ is to allocate all the power to jammer node $b$.

Proof: In Proposition 4.1, consider $f[i]$ as $f[i]=\delta[i-k]$, where $\delta[\cdot]$ denotes the unit sample (impulse) function; hence, the corresponding $v[i]$ is given by $v[i]=\lambda g[i]+(1-\lambda) \delta[i-k]$. 
If the inequality $P_{T} \leq P_{T}^{(k)}$ holds, then the condition in (23) is satisfied by $f[i]$, according to Proposition 4 in [13], in which case the power vector $\boldsymbol{p}_{*}^{J}$ that allocates all the power to jammer node $b$ is the solution of Scheme 2. On the other hand, the power vector $\boldsymbol{p}_{*}^{J}$, which is the solution of Scheme 2 and satisfies (23), is the solution of Scheme 1 for $v[i]$ if the index of the first largest element of vector $\boldsymbol{w}=\sum_{i=1}^{N_{T}} v[i] \boldsymbol{d}_{i}$ is $b$, which occurs when the following condition holds:

$$
b=\underset{n \in\left\{1,2, \ldots, N_{J}\right\}}{\arg \max }\left(\lambda \sum_{i=1}^{N_{T}} g[i] r_{i}\left|\gamma_{i n}\right|^{2}+(1-\lambda) r_{k}\left|\gamma_{k n}\right|^{2}\right) .
$$

Since the expression in (35) is an affine function of $\lambda$ for each $n \in\left\{1,2, \ldots, N_{J}\right\}$ and $b=\arg \max _{n \in\left\{1,2, \ldots, N_{J}\right\}} r_{k}\left|\gamma_{k n}\right|^{2}$, the $b$ th index of $\boldsymbol{w}$ has the highest value until the first intersection of the line corresponding to $b$ and any of the remaining lines corresponding to $n \in\left\{1,2, \ldots, N_{J}\right\} \backslash b$. Therefore, if $0 \leq \lambda \leq$ $\tilde{\zeta}$, where $\tilde{\zeta}$ is given by (33), then the solution of Scheme 1 for $v[i]$ is to allocate all the power to jammer node $b$ corresponding to the largest element of $\boldsymbol{w}$. This implies, according to Proposition 4.1, that the solution that assigns all the power to jammer node $b$ is also the solution of the restricted scheme. Hence, allocating all the power to jammer node $b$ is the solution for both the restricted scheme and Scheme 2 for $0 \leq \lambda \leq \tilde{\zeta}$ if $P_{T} \leq P_{T}^{(k)}$.

Corollary 4.5 implies that if $\tilde{\zeta} \geq 1$, then the restricted scheme and Scheme 2 are identical for any value of the design parameter $\lambda$ in (22) if $P_{T} \leq P_{T}^{(k)}$. It is concluded from (31), (33), and (34) that $\tilde{\zeta} \geq 1$ occurs when $b=$ $\arg \max _{n \in\left\{1,2, \ldots, N_{J}\right\}} \sum_{i=1}^{N_{T}} g[i] r_{i}\left|\gamma_{i n}\right|^{2}$.

In the following proposition (which is based on Proposition 5 in [13] and the minimax theorem), the numbers of target and jammer nodes which are effective in the solution of the restricted scheme (i.e., (22)) are specified. Proposition 5 in [13] presents only the number of jammer nodes that determine the solution of Scheme 2. Based on the minimax theorem, the following proposition enhances this property by also specifying the number of target nodes that are involved in the solution of the restricted scheme.

Proposition 4.6: Suppose that $r_{i}$ defined in (14) is finite for each $i$. Then, in the absence of peak power constraints, the solution of the restricted scheme is determined by at most $N_{T}$ jammer nodes, where $N_{T}$ is the number of target nodes. In addition, $f[i]$ in Proposition 4.1 contains at most $N_{J}$ point masses, which implies that the solution of Scheme 2 (i.e., the restricted scheme for $\lambda=0$ ) is determined by at most $N_{J}$ target nodes, where $N_{J}$ is the number of jammer nodes.

Proof: In the absence of the peak power constraint, (22c) becomes ineffective; hence, (22) can be reformulated as follows:

$$
\operatorname{maximize}_{\tilde{\boldsymbol{p}}^{J} \in \mathcal{A}_{N_{J}}} \min _{\tilde{\boldsymbol{f}} \in \mathcal{A}_{N_{T}}} P_{T}(\lambda \boldsymbol{g}+(1-\lambda) \tilde{\boldsymbol{f}})^{T} \boldsymbol{D} \tilde{\boldsymbol{p}}^{J}
$$

where $\tilde{\boldsymbol{p}}^{J} \triangleq \boldsymbol{p}^{J} / P_{T}, \mathbf{1}^{T} \tilde{\boldsymbol{p}}^{J}=1$, and $\tilde{\boldsymbol{p}}^{J} \succeq \mathbf{0} ; \mathbf{1}^{T} \tilde{\boldsymbol{f}}=1$ and $\tilde{\boldsymbol{f}} \succeq \mathbf{0} ; \boldsymbol{D} \triangleq\left[\boldsymbol{d}_{1} \boldsymbol{d}_{2} \cdots \boldsymbol{d}_{N_{T}}\right]^{T}$; and finally $\boldsymbol{g} \triangleq[g[1] g[2] \cdots$ $\left.g\left[\bar{N}_{T}\right]\right]^{T}$. From the foregoing constraints on $\tilde{\boldsymbol{p}}^{J}$ and $\tilde{\boldsymbol{f}}$, it is clear that set $\mathcal{A}_{m}$ is given by

$$
\mathcal{A}_{m}=\left\{\boldsymbol{h} \in \mathbb{R}^{m} \mid \boldsymbol{h} \succeq \mathbf{0} \text { and } \mathbf{1}^{T} \boldsymbol{h}=1\right\}
$$

for $m \in\left\{N_{J}, N_{T}\right\}$, which is compact and convex. It should also be noted that the objective function in (36) is an affine function of $\tilde{\boldsymbol{f}}$ for a fixed $\tilde{\boldsymbol{p}}^{J}$, and a linear function of $\tilde{\boldsymbol{p}}^{J}$ for a fixed $\tilde{f}$. Hence, the minimax theorem [34] can be applied to (36), implying that (36) is equivalent to the following optimization problem:

$$
\underset{\tilde{\boldsymbol{f}} \in \mathcal{A}_{N_{T}}}{\operatorname{minimize}} \max _{\tilde{\boldsymbol{p}}^{J} \in \mathcal{A}_{N_{J}}} P_{T}(\lambda \boldsymbol{g}+(1-\lambda) \tilde{\boldsymbol{f}})^{T} \boldsymbol{D} \tilde{\boldsymbol{p}}^{J}
$$

which can also be expressed as follows:

$$
\underset{\tilde{\boldsymbol{f}} \in \mathcal{A}_{N_{T}}}{\operatorname{minimize}} \max _{\tilde{\boldsymbol{p}}^{J} \in \mathcal{A}_{N_{J}}}\left(\tilde{\boldsymbol{p}}^{J}\right)^{T} \boldsymbol{D}^{T}(\lambda \boldsymbol{g}+(1-\lambda) \tilde{\boldsymbol{f}}) P_{T} .
$$

For a given $\tilde{\boldsymbol{p}}^{J} \in \mathcal{A}_{N_{J}}$, the objective function in (36) is minimized for $\tilde{\boldsymbol{f}}=\boldsymbol{e}_{k}$, where $k=\arg \min _{i \in\left\{1,2, \ldots, N_{T}\right\}} \boldsymbol{d}_{i}^{T} \tilde{\boldsymbol{p}}^{J}$ and $e_{i}$ denotes the vector whose $i$ th element is 1 and other elements are 0 . Hence, the possible set of values that can be assumed by $\tilde{f}$ in (36) contains only $N_{T}$ elements. In a similar way, the maximizer of the objective function in (39) for a given $\tilde{f} \in \mathcal{A}_{N_{T}}$ is provided by a vector of the form $\boldsymbol{e}_{i}$, which confines the number of possible values of $\tilde{\boldsymbol{p}}^{J}$ to $N_{J}$. Therefore, applying the steps followed in the proof of Proposition 5 in [13] to (36), it is concluded that the solution of the restricted scheme is determined by at most $N_{T}$ jammer nodes. Similarly, applying those same steps to (39), it is concluded that $f[i]$ in Proposition 4.1 contains at most $N_{J}$ point masses, which also implies that the solution of the restricted scheme for $\lambda=0$ (i.e., Scheme 2) is determined by at most $N_{J}$ target nodes. (When $\lambda=0$, the solution of Scheme 1 for $v[i]=f[i]$ is also the solution of the restricted scheme, which is identical to Scheme 2.)

Proposition 4.6 yields an essential result for determining the optimal jammer power allocation strategies under the restricted scheme. It is noted from Proposition 4.6 that the optimal jammer power vector contains at most $N_{T}$ non-zero elements, which facilitates the elimination of the solutions that allocate nonzero power to more than $N_{T}$ jammer nodes. Hence, the solution space for power vectors is significantly reduced based on Proposition 4.6, especially for scenarios where the number of target nodes is considerably lower than that of jammer nodes. In addition, the set of PMFs $f[i]$ that satisfy the conditions in Proposition 4.1 is reduced by constraining the search for feasible PMFs to only those having at most $N_{J}$ non-zero elements. Therefore, for wireless network configurations containing fewer jammer nodes than target nodes (for which Proposition 5 in [13] does not help reduce the solution space of Scheme 2), Proposition 4.6 facilitates the solution of the restricted scheme, which relies on finding a PMF $f[i]$ as defined in Proposition 4.1. Furthermore, the solution of Scheme 2 (the restricted scheme for $\lambda=0$ ) is also the solution of Scheme 1 for $f[i]$, where $f[i]$ satisfies the condition in (23). Hence, based on Remark 5, it is stated that the least-favorable PMF contains at most $N_{J}$ point masses. 


\section{EXTENSION OF RESTRICTED SCHEME}

In this section, the restricted scheme is extended to cover more generic scenarios by utilizing the framework in [25]. Based on their significance levels, the target nodes can be grouped into subsets $\Lambda_{1}, \ldots, \Lambda_{M}$ with a decreasing order of significance levels (i.e., subset $\Lambda_{1}$ contains the target nodes with the highest significance levels), where $\Lambda_{1} \subset \Lambda_{2} \subset \cdots \subset \Lambda_{M}=$ $\left\{1,2, \ldots, N_{T}\right\}$. Then, the restricted scheme formulation in (21) can be extended as follows [25]:

$$
\begin{array}{ll}
\underset{\boldsymbol{p}^{J}}{\operatorname{maximize}} & C_{\mathrm{avg}}\left(\boldsymbol{p}^{J}\right) \\
\text { subject to } & \min _{i \in \Lambda_{k}} C_{i}\left(\boldsymbol{p}^{J}\right) \geq \alpha_{k}, k=1, \ldots, M \\
& \mathbf{1}^{T} \boldsymbol{p}^{J}=P_{T} \\
& 0 \leq P_{\ell}^{J} \leq P_{\ell}^{\text {peak }}, \ell=1,2, \ldots, N_{J}
\end{array}
$$

where $\alpha_{1}>\cdots>\alpha_{M}$ are the design parameters. The formulation in (40) corresponds to an LP problem, as well. In addition, it can be shown that the full total power utilization property also holds for this extended scheme (cf. Lemma 3 in [13]). The following proposition characterizes the solution of (40), which can be obtained by following the steps in the proofs of Proposition 4.1 in Section IV and Theorem 4 in [25]:

Proposition 5.1: Define a PMF $v[i]$ as $v[i] \triangleq \lambda_{0} g[i]+$ $\sum_{k=1}^{M} \lambda_{k} f_{k}[i]$ with $\lambda_{k} \geq 0$ and $\sum_{k=0}^{M} \lambda_{k}=1$, where $f_{k}[i]$ is any valid PMF defined over set $\Lambda_{k}$. If $\boldsymbol{p}_{*}^{J}$ is the solution of Scheme 1 for $v[i]$ and satisfies

$$
\sum_{i \in \Lambda_{k}} f_{k}[i] C_{i}\left(\boldsymbol{p}_{*}^{J}\right)=\min _{i \in \Lambda_{k}} C_{i}\left(\boldsymbol{p}_{*}^{J}\right)=\alpha_{k}, k=1, \ldots, M
$$

then it solves the optimization problem in (40).

\section{Alternative Scheme}

The general objective of jammer networks is to degrade the localization performance of target nodes with respect to a performance measure. In Section III and Section IV, optimal jammer power allocation strategies have been investigated to maximize a certain function of the CRLBs of target nodes. However, for some localization systems, it may be more critical to maximize the number of disabled (deactivated) target nodes than to maximize their average or minimum CRLB, where a target node is assumed to be disabled when its CRLB exceeds a predefined level (i.e., when its localization accuracy becomes useless for the considered application). For instance, in military applications, the effectiveness of the jamming system may depend on the number of deactivated enemy targets. Therefore, in this section, a new scheme is proposed with the aim of maximizing the number of disabled target nodes in a wireless localization system under average and peak power constraints. The proposed power allocation scheme optimizes a jamming performance metric that is different from the one in (21) and can be more critical in practice for certain localization scenarios. Let $\Lambda$ denote any subset of $\left\{1,2, \ldots, N_{T}\right\}$, and $|\Lambda|$ represent the size of subset $\Lambda$. Then, the proposed scheme is formulated as follows:

$$
\begin{aligned}
\underset{\boldsymbol{p}^{J}}{\operatorname{maximize}} & |\Lambda| \\
\text { subject to } & \min _{i \in \Lambda} C_{i}\left(\boldsymbol{p}^{J}\right) \geq t \\
& \mathbf{1}^{T} \boldsymbol{p}^{J} \leq P_{T} \\
& 0 \leq P_{\ell}^{J} \leq P_{\ell}^{\text {peak }}, \ell=1,2, \ldots, N_{J} \\
& \Lambda \subseteq\left\{1,2, \ldots, N_{T}\right\}
\end{aligned}
$$

where $t$ is the predefined level for disabling a target node (i.e., a target node with a CRLB larger than $t$ is regarded as disabled).

For convenience, let the resulting minimum CRLB of the target nodes in a network operating according to Scheme 2 be named as the max-min CRLB in accordance with the formulation of Scheme 2 in (20). The following definition introduces a new parameter that will facilitate the solution of (41).

Definition 6.1: Let $\Lambda_{k}$ with $k \in\left\{1,2, \ldots, N_{T}\right\}$ be the subset consisting of $k$ target nodes which constitute the subnetwork achieving the maximum max-min CRLB among all possible subnetworks with $k$ target nodes. Then, $t_{k}$ is defined as the corresponding max-min CRLB.

Based on Definition 6.1, it should be noted that $t_{1}$ is the maximum achievable CRLB for an individual target node in the whole network; hence, $t_{1}$ is the maximum value which $t$ can take in practice (i.e., the maximum number of disabled target nodes is zero when $t>t_{1}$ ). The following corollary demonstrates the monotonicity of $t_{k}$ with respect to the number of target nodes $k$.

Corollary 6.2: For $k \in\left\{1,2, \ldots, N_{T}-1\right\}, t_{k}$ is larger than or equal to $t_{k+1}$, where $t_{k}$ is as defined in Definition 6.1.

Proof: Let $\mathcal{S}_{k}$ be the set of all $k$-element subsets of $\left\{1,2, \ldots, N_{T}\right\}$. Then, assuming that the power vector $\boldsymbol{p}^{J}$ satisfies the total and peak power constraints,

$$
\begin{aligned}
t_{k+1} & =\max _{\Gamma \in \mathcal{S}_{k+1}} \max _{\boldsymbol{p}^{J}} \min _{i \in \Gamma} C_{i}\left(\boldsymbol{p}^{J}\right) \\
& =\min _{i \in \Lambda_{k+1}} C_{i}\left(\boldsymbol{p}_{*}^{J}\right) \\
& \leq \max _{\Gamma \in \mathcal{G}_{k}} \min _{i \in \Gamma} C_{i}\left(\boldsymbol{p}_{*}^{J}\right) \\
& \leq \max _{\Gamma \in \mathcal{G}_{k}} \max _{\boldsymbol{p}^{J}} \min _{i \in \Gamma} C_{i}\left(\boldsymbol{p}^{J}\right) \\
& \leq \max _{\Gamma \in \mathcal{S}_{k}} \max _{\boldsymbol{p}^{J}} \min _{i \in \Gamma} C_{i}\left(\boldsymbol{p}^{J}\right)=t_{k}
\end{aligned}
$$

where $\boldsymbol{p}_{*}^{J}$ is the optimal power vector in (42) and $\mathcal{G}_{k}$ denotes the set of all $k$-element subsets of $\Lambda_{k+1}$.

Next, the following proposition specifies the solution of (41):

Proposition 6.3: In the case of $t_{k+1}<t \leq t_{k}$ for $k=$ $1,2, \ldots, N_{T}$ (where $t_{N_{T}+1}$ is defined as zero), the solution of (41) is the same as that of Scheme 2 obtained for the subnetwork consisting of all the target nodes in subset $\Lambda_{k}$, and the corresponding number of disabled target nodes is equal to $k$.

Proof: Consider the case of $t_{k+1}<t \leq t_{k}$. The proof is based on showing that the maximum value of $|\Lambda|$ in (41) must be smaller than $k+1$ and that there exists a subset $\Lambda$ consisting of $k$ target nodes which satisfy the constraints in (41). Assume 
that the size of subset $\Lambda$ is given to be equal to $k+1$. Then, based on the definitions of $\Lambda_{k+1}$ and $t_{k+1}$, it is concluded that the maximum value the minimum CRLB $\min _{i \in \Lambda} C_{i}\left(p^{J}\right)$ can take is equal to $t_{k+1}$, which is achieved by $\boldsymbol{p}^{J}$ corresponding to the solution of Scheme 2 obtained for the subnetwork consisting of all the target nodes in subset $\Lambda_{k+1}$. This means that the size of $\Lambda$ must be lower than $k+1$ in order for $\min _{i \in \Lambda} C_{i}\left(\boldsymbol{p}^{J}\right) \geq t$ to hold. Next, consider subset $\Lambda_{k}$ whose size is $k$ by definition. Then, from the definitions of $\Lambda_{k}$ and $t_{k}$, the minimum CRLB $\min _{i \in \Lambda_{k}} C_{i}\left(\boldsymbol{p}^{J}\right)$ achieves $t_{k}$ with $\boldsymbol{p}^{J}$ corresponding to the solution of Scheme 2 obtained for the subnetwork consisting of all the target nodes in subset $\Lambda_{k}$.

It is noted that the solution of (41) does not have to be unique; that is, there can be more than one solution for the problem in (41). In Proposition 6.3, only one of the solutions is specified.

\section{Simulation RESUlts}

In this section, the proposed schemes are investigated through simulations. Parameter $\lambda_{i j}$ in (9) is computed by $\lambda_{i j}=100 N_{0}\left\|\boldsymbol{x}_{i}-\boldsymbol{y}_{j}\right\|^{-2} / 2$ based on the free space propagation model presented in [15], and the peak power limits are assigned as $P_{\ell}^{\text {peak }}=10, \forall \ell$. Also, $\left|\gamma_{i \ell}\right|^{2}$ in (10) is modeled as $\left|\gamma_{i \ell}\right|^{2}=\left\|\boldsymbol{x}_{i}-\boldsymbol{z}_{\ell}\right\|^{-2}$, and the total power $P_{T}$ is normalized as $\bar{P}_{T}=2 P_{T} / N_{0}$ [14]. In addition, $N_{0}$ is taken as 2 , and LOS connections to all the anchor nodes are assumed for each target node.

In the following subsections, three different localization scenarios are considered to corroborate the theoretical derivations obtained for the proposed optimization schemes. Specifically, we provide examples for the restricted scheme properties in Proposition 4.1, Corollary 4.2, Proposition 4.4, Corollary 4.5, and Proposition 4.6, and for the alternative scheme properties in Corollary 6.2 and Proposition 6.3. The first scenario includes a network configuration where jammer nodes are placed inside the convex hull of anchor nodes. In the second scenario, jammer nodes are located outside the convex hull of anchor nodes to explore the theoretical results under various network configurations. Finally, the purpose of the third scenario is to validate the sparsity property of the optimal power allocation vector as demonstrated in Proposition 4.6.

\section{A. Scenario 1: Jammers Inside the Convex Hull of Anchors}

For the first set of simulations, a network consisting of six anchor nodes, five target nodes, and three jammer nodes is considered, where the node locations are as illustrated in Fig. 1. The PMF $g[i]$ is defined as $g[1]=0.4, g[2]=g[4]=0.1$, and $g[3]=g[5]=0.2$.

1) Restricted Scheme: In Fig. 2, the average CRLB of the restricted scheme is plotted against $\alpha$ for various values of the normalized total power $\bar{P}_{T}$, where $\alpha \in[\alpha, \bar{\alpha}]$. It is observed that the average CRLB is a strictly decreasing and concave function of $\alpha$ for $\alpha \in[\underline{\alpha}, \bar{\alpha}]$ in accordance with Proposition 4.4. Also, the edge points of the curves in Fig. 2 are marked as Scheme 1 and Scheme 2 since the restricted scheme converges to Scheme 1 for $\alpha=\underline{\alpha}$ and to Scheme 2 for $\alpha=\bar{\alpha}$ (see the paragraph before Proposition 4.4). Moreover, the average CRLB achieved by the restricted scheme and the values of $\underline{\alpha}$ and $\bar{\alpha}$ become larger as the

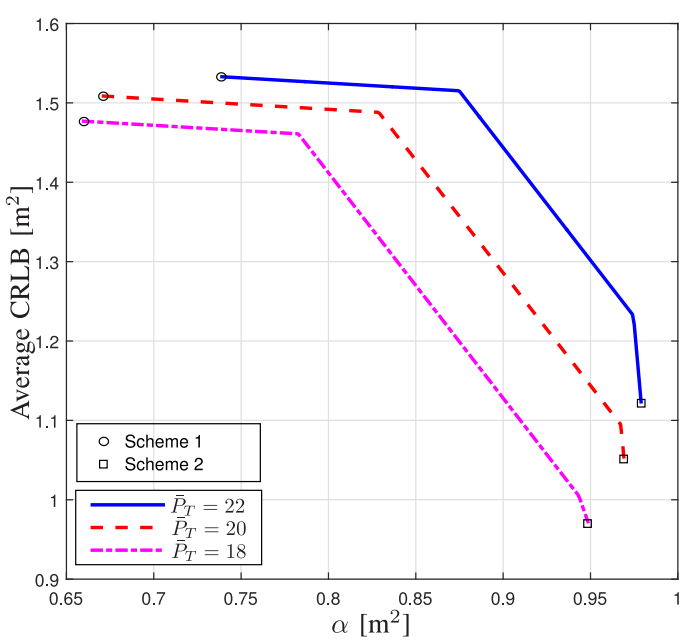

Fig. 2. Average CRLB versus $\alpha$ for the restricted schemes corresponding to $\bar{P}_{T}=22, \bar{P}_{T}=20$, and $\bar{P}_{T}=18$ for the scenario in Fig. 1 , where $\alpha \in[\underline{\alpha}, \bar{\alpha}]$. The edge points marked by circles and squares correspond to Scheme 1 and Scheme 2, respectively.

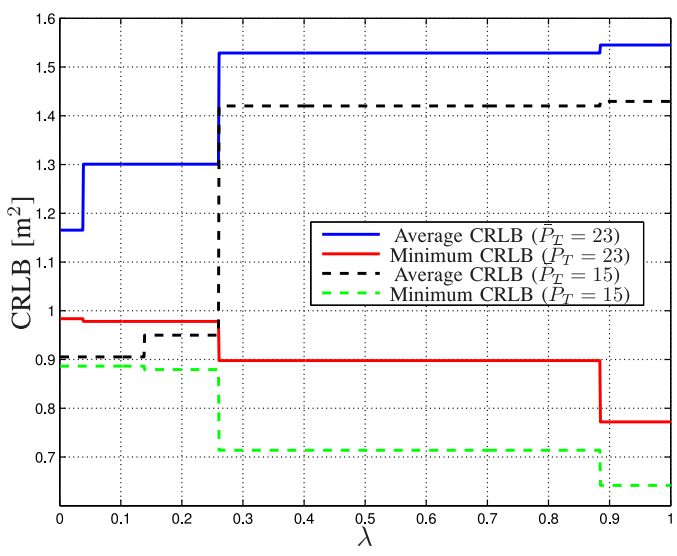

Fig. 3. Average and minimum CRLBs versus $\lambda$ for the restricted schemes corresponding to $\bar{P}_{T}=23$ and $\bar{P}_{T}=15$ for the scenario in Fig. 1 .

total power increases, which agrees with the CRLB expression in (13) and the definitions of $\underline{\alpha}$ and $\bar{\alpha}$.

In Fig. 3, the average and minimum CRLBs of the restricted scheme are illustrated versus $\lambda$ for various values of the normalized total power $\bar{P}_{T}$. From the figure, it is seen that an increase in $\lambda$ can result in an increase in the average CRLB and a decrease in the minimum CRLB, which means that as $\lambda$ increases the restricted scheme converges to Scheme 1 whereas it converges to Scheme 2 as $\lambda$ decreases; this is in fact the role assigned to $\lambda$, which is specified by (22). In addition, it is observed that larger average and minimum CRLBs can be attained for higher values of the total power, as implied by the CRLB expression in (13). Furthermore, it is noted from Fig. 3 that there can exist discontinuities in the average and minimum CRLBs, which occur due to the changes in the nature of the optimal power allocation strategies of the jammer nodes. However, it can be shown that the objective function in (22) (which is the combination of the average and the minimum CRLBs) is continuous with respect to $\lambda$. 


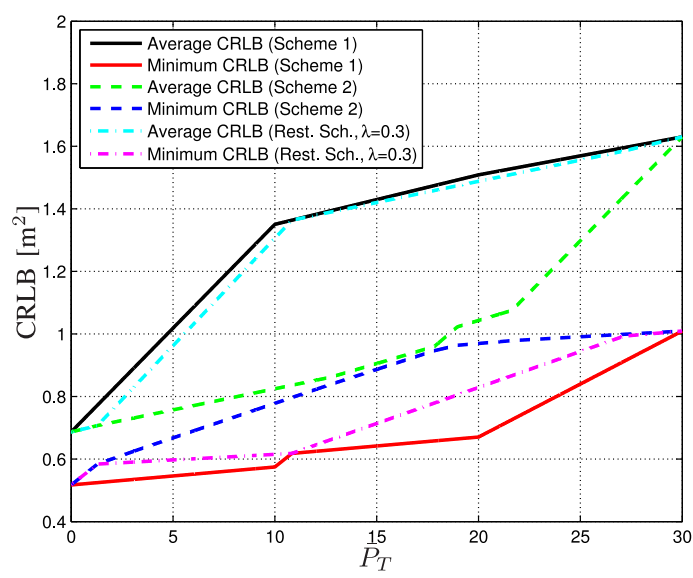

Fig. 4. Average and minimum CRLBs of Scheme 1, Scheme 2, and the restricted scheme (for $\lambda=0.3$ ) versus $\bar{P}_{T}$ for the scenario in Fig. 1.

TABLE I

Allocated POWERS to JAMMER NODES FOR THE RESTRICTED SCHEME FOR THE SCENARIO IN FIG. 1

\begin{tabular}{|l|lll|lll|}
\hline \multirow{2}{*}{$\bar{P}_{T}$} & \multicolumn{4}{|c|}{$\lambda=0.2$} & \multicolumn{3}{c|}{$\lambda=0.6$} \\
\cline { 2 - 7 } & $P_{1}^{J}$ & $P_{2}^{J}$ & $P_{3}^{J}$ & $P_{1}^{J}$ & $P_{2}^{J}$ & $P_{3}^{J}$ \\
\hline 3 & 0 & 1.0488 & 1.9512 & 3 & 0 & 0 \\
\hline 6 & 0.2533 & 2.7419 & 3.0049 & 6 & 0 & 0 \\
\hline 9 & 0.5283 & 4.4222 & 4.0495 & 9 & 0 & 0 \\
\hline 12 & 0.8033 & 6.1025 & 5.0942 & 10 & 0.7117 & 1.2883 \\
\hline 15 & 1.0783 & 7.7828 & 6.1389 & 10 & 2.5532 & 2.4468 \\
\hline 18 & 1.3533 & 9.4631 & 7.1836 & 10 & 4.3947 & 3.6053 \\
\hline 21 & 3.5789 & 10 & 7.4211 & 10 & 6.2362 & 4.7638 \\
\hline 24 & 6.7206 & 10 & 7.2794 & 10 & 8.0777 & 5.9223 \\
\hline 27 & 9.8622 & 10 & 7.1378 & 10 & 9.9192 & 7.0808 \\
\hline
\end{tabular}

In Fig. 4, the average and minimum CRLBs of Scheme 1, Scheme 2, and the restricted scheme (for $\lambda=0.3$ ) are plotted versus the normalized total power. The figure emphasizes the main characteristic of the restricted scheme; namely, the restricted scheme can provide a trade-off between Scheme 1 and Scheme 2: An increase in the minimum CRLB can be provided at the expense of a decrease in the average CRLB, or vice versa. Also, in compliance with the definitions of Scheme 1, Scheme 2, and the restricted scheme, the maximum and the minimum gaps between the average and the minimum CRLBs are, respectively, achieved by Scheme 1 and Scheme 2 for all $\bar{P}_{T}$. Thus, it can be concluded that the restricted scheme trades off the complexity for a decrease or an increase in the gap between the average and the minimum CRLBs. Furthermore, $P_{T}^{(k)}$ and $\tilde{\zeta}$ in Corollary 4.5 are calculated as $P_{T}^{(k)}=1.2914$ and $\tilde{\zeta}=0.4761$, which, according to Corollary 4.5 , indicates that the restricted scheme and Scheme 2 are identical for $P_{T} \leq 1.2914$ and $\lambda \leq 0.4761$. As observed from Fig. 4, the restricted scheme for $\lambda=0.3$ and Scheme 2 achieve the same minimum CRLB for $P_{T} \leq 1.2914$, which complies with Corollary 4.5. In Table I, the optimal power allocation strategies corresponding to the restricted schemes (for $\lambda=0.2$ and $\lambda=0.6$ ) are presented for various values of $\bar{P}_{T}$ for the scenario in Fig. 1. It is observed that the optimal power allocation strategy can assign power to one, two, or all three jammer node(s) in different scenarios. In addition, it is observed that as the total power limit increases further, the peak power

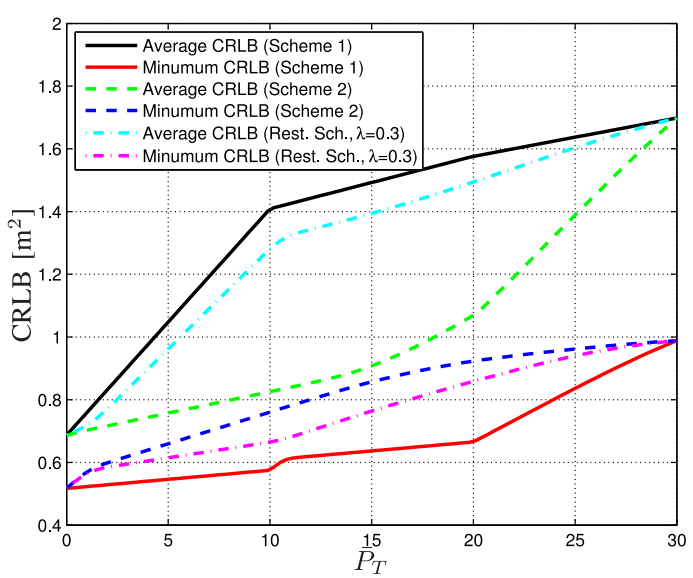

Fig. 5. Average and minimum CRLBs of Scheme 1, Scheme 2, and the restricted scheme (for $\lambda=0.3$ ) versus $\bar{P}_{T}$ for the scenario in Fig. 1 with jammer nodes uniformly distributed over a disk whose boundary is represented by dashed lines in Fig. 1.

limit starts becoming effective. As a result, it is noted that the optimal jamming policy may vary depending on the specific network configuration, the choice of the design parameter $\lambda$ in (22a), the total power limit in (22b), and the peak power limit in $(22 \mathrm{c})$.

In order to derive the mean jamming performance when the jammer nodes are randomly placed over an area of interest, simulations are carried out for the network in Fig. 1. In the simulations, the anchor and target nodes have fixed positions whereas the jammer nodes are uniformly distributed over a disk centered at the original jammer positions with a radius of $1 \mathrm{~m}$, as shown in Fig. 1. Fig. 5 illustrates the average and the minimum CRLBs averaged over 1000 Monte Carlo cycles for Scheme 1, Scheme 2, and the restricted scheme for $\lambda=0.3$. Similar to the fixed configuration used in Fig. 4, Fig. 5 highlights the compromising nature of the restricted scheme. In addition, it is observed that random deployment of jammer nodes serves as a smoothing mechanism for the CRLB curves of all the schemes due to the averaging effect.

2) Alternative Scheme: The alternative scheme proposed in Section VI is investigated in Fig. 6 for the scenario in Fig. 1. The figure plots the number of disabled target nodes versus $t$ for various values of normalized total power $\bar{P}_{T}$. It is observed from the figure that the number of disabled target nodes decreases as $t$ increases, which is in agreement with Proposition 6.3. Also, the jamming performance measured with respect to the alternative scheme in (41) can be improved by increasing the total power, which is highly intuitive. For $\bar{P}_{T}=11$, it is calculated that $\Lambda_{4}=$ $\{1,2,4,5\}, \Lambda_{3}=\{1,3,5\}, \Lambda_{2}=\{1,3\}$ and $\Lambda_{1}=\{1\}$; and for $\bar{P}_{T}=22$, it is calculated that $\Lambda_{4}=\{1,2,3,5\}, \Lambda_{3}=\{1,3,5\}$, $\Lambda_{2}=\{1,3\}$ and $\Lambda_{1}=\{1\}$. In Table II, the optimal power allocation strategies for the alternative scheme are presented along with the corresponding values of $t_{k}$ for various values of $\bar{P}_{T}$ for the scenario in Fig. 1. It is noted that some jammer nodes are ineffective in certain scenarios (e.g., for $k=1,2,5$ and $\bar{P}_{T}=11$ ) while all the jammer nodes are assigned nonzero powers in other scenarios (e.g., for $k=3,4$ and $\bar{P}_{T}=11$ ). In addition, 


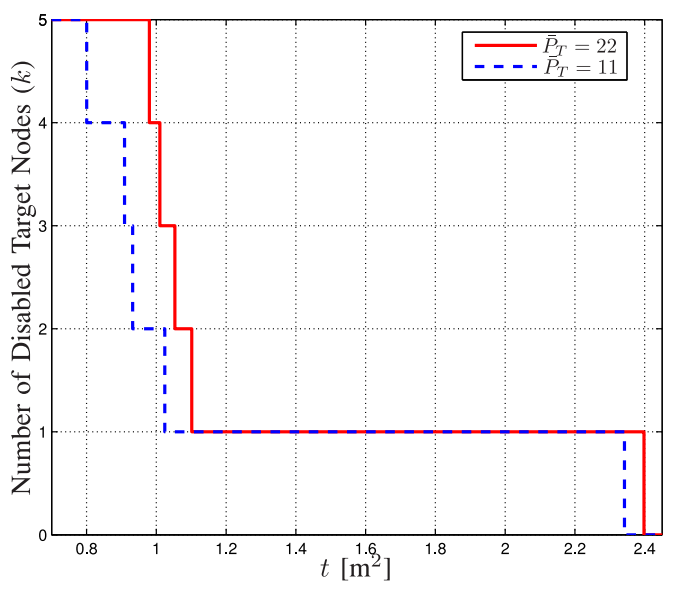

Fig. 6. Alternative schemes (for $\bar{P}_{T}=11$ and $\bar{P}_{T}=22$ ) versus $t$ for the scenario in Fig. 1.

TABLE II

PARAMETER $t_{k}$ AND Allocated POWERS TO JAMMER NODES FOR THE ALTERNATIVE SCHEME FOR THE SCENARIO IN FIG. 1

\begin{tabular}{|l|llll|llll|}
\hline \multirow{3}{*}{$k$} & \multicolumn{3}{|c|}{$\bar{P}_{T}=11$} & \multicolumn{4}{c|}{$\bar{P}_{T}=22$} \\
\cline { 2 - 9 } & $t_{k}$ & $P_{1}^{J}$ & $P_{2}^{J}$ & $P_{3}^{J}$ & $t_{k}$ & $P_{1}^{J}$ & $P_{2}^{J}$ & $P_{3}^{J}$ \\
\hline 5 & 0.8002 & 0 & 6.1117 & 4.8883 & 0.9799 & 2 & 10 & 10 \\
\hline 4 & 0.9088 & 0.4782 & 9.7938 & 0.7280 & 1.0103 & 3.8493 & 10 & 8.1507 \\
\hline 3 & 0.9319 & 0.7288 & 0.2712 & 10 & 1.0533 & 2 & 10 & 10 \\
\hline 2 & 1.0242 & 1.3392 & 0 & 9.6608 & 1.1015 & 10 & 2 & 10 \\
\hline 1 & 2.3421 & 10 & 1 & 0 & 2.3980 & 10 & 10 & 2 \\
\hline
\end{tabular}

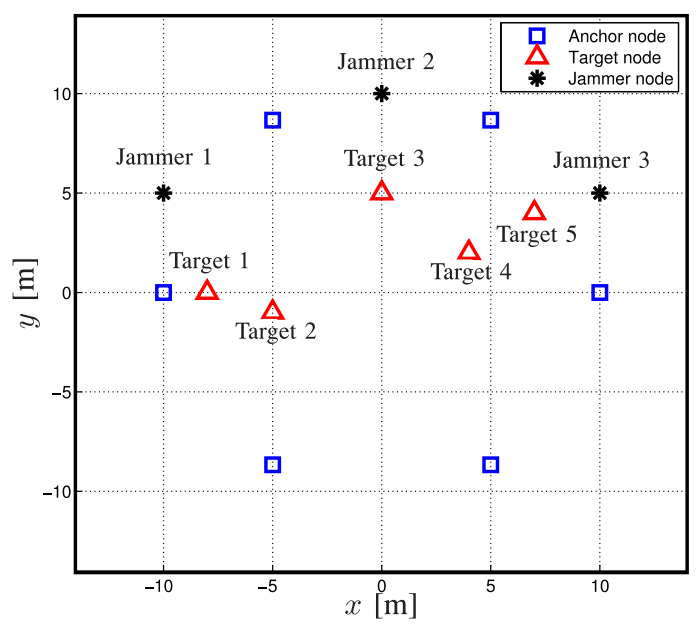

Fig. 7. The network considered in the simulations, where the anchor node positions are $[-100],[-5-5 \sqrt{3}],[-55 \sqrt{3}],[55 \sqrt{3}],[5-5 \sqrt{3}]$, and $[100] \mathrm{m}$., the target node positions are $[-80],[-5-1],[05],[42]$ and $[74] \mathrm{m}$., and the jammer node positions are $[-105],[010]$, and $[105] \mathrm{m}$.

the monotonicity property of $t_{k}$ with respect to $k$, as shown in Corollary 6.2, can be seen from Table II.

\section{B. Scenario 2: Jammers Outside the Convex Hull of Anchors}

Secondly, the network illustrated in Fig. 7 is considered. Unlike the previous scenario, the jammer nodes are located outside the convex hull of the anchor nodes. The PMF $g[i]$ for this scenario is defined as $g[1]=g[2]=0.1, g[3]=g[4]=0.2$, and $g[5]=0.4$.

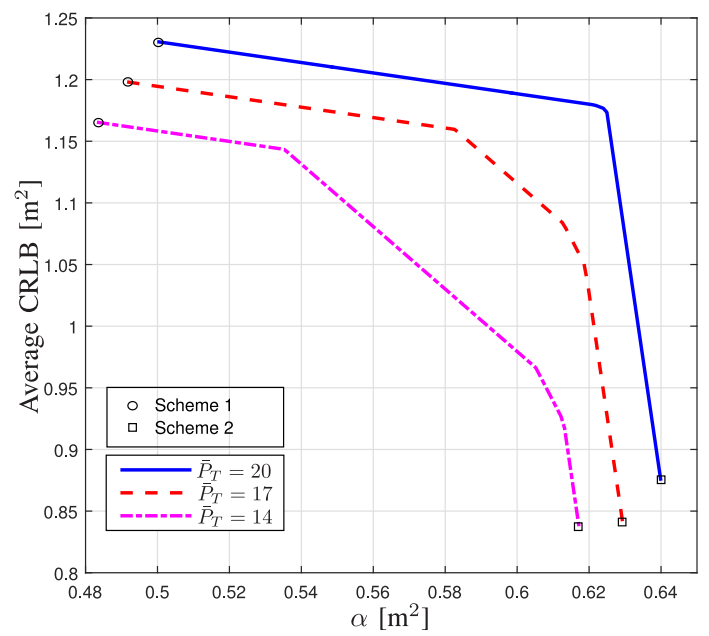

Fig. 8. Average CRLB versus $\alpha$ for the restricted schemes corresponding to $\bar{P}_{T}=20, \bar{P}_{T}=17$ and $\bar{P}_{T}=14$ for the scenario in Fig. 7 . The edge points marked by circles and squares correspond to Scheme 1 and Scheme 2, respectively.

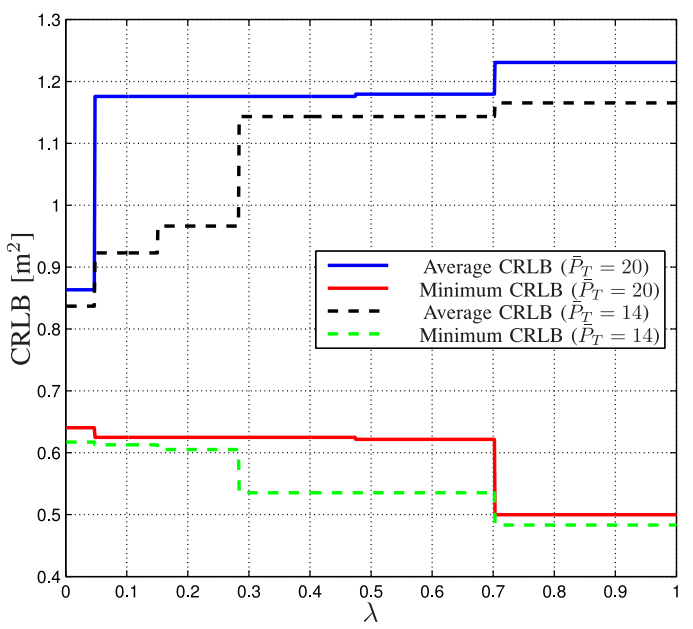

Fig. 9. Average and minimum CRLBs versus $\lambda$ for the restricted schemes corresponding to $\bar{P}_{T}=20$ and $\bar{P}_{T}=14$ for the scenario in Fig. 7 .

1) Restricted Scheme: In Fig. 8, the average CRLB versus $\alpha$ is plotted for the restricted scheme for various values of the normalized total power $\bar{P}_{T}$. In Fig. 9, the average and the minimum CRLBs of the restricted scheme are illustrated against $\lambda$ for various values of the normalized total power $\bar{P}_{T}$. In Fig. 10, the average and the minimum CRLBs of Scheme 1, Scheme 2, and the restricted scheme (for $\lambda=0.5$ ) are illustrated versus the normalized total power. From the figure, it is observed that the restricted scheme (for $\lambda=0.5$ ) is identical to Scheme 2 for $\bar{P}_{T} \leq 9.75$ and identical to Scheme 1 for $\bar{P}_{T} \geq 19.28$. For all of these figures, similar observations to those for the network in Fig. 1 are made. Table III presents the optimal power allocation strategies corresponding to the restricted schemes (for $\lambda=0.2$ and $\lambda=0.8$ ) for various values of $\bar{P}_{T}$ for the scenario in Fig. 7. The values of $P_{T}^{(k)}, \tilde{\zeta}$, and $b$ in Corollary 4.5 are calculated as $P_{T}^{(k)}=7.3787$, $\tilde{\zeta}=0.2830$, and $b=1$. As seen from Table III, the optimal strategy for $\lambda=0.2$ is to allocate all the power to jammer node 


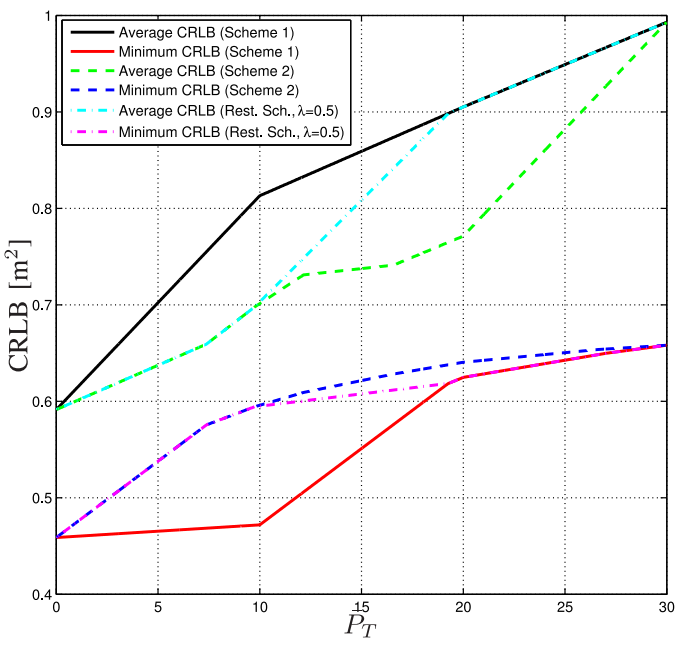

Fig. 10. Average and minimum CRLBs of Scheme 1, Scheme 2, and the restricted scheme (for $\lambda=0.5$ ) versus $\bar{P}_{T}$ for the scenario in Fig. 7.

TABLE III

AllocAted POWERS TO JAMMER NODES FOR THE RESTRICTED SCHEME FOR THE SCENARIO IN FIG. 7

\begin{tabular}{|l|lll|lll|}
\hline \multirow{2}{*}{$\bar{P}_{T}$} & \multicolumn{3}{|c|}{$\lambda=0.2$} & \multicolumn{3}{c|}{$\lambda=0.8$} \\
\cline { 2 - 7 } & $P_{1}^{J}$ & $P_{2}^{J}$ & $P_{3}^{J}$ & $P_{1}^{J}$ & $P_{2}^{J}$ & $P_{3}^{J}$ \\
\hline 3 & 3 & 0 & 0 & 0 & 0 & 3 \\
\hline 6 & 6 & 0 & 0 & 0 & 0 & 6 \\
\hline 9 & 8.1195 & 0 & 0.8805 & 0 & 0 & 9 \\
\hline 12 & 9.9048 & 0 & 2.0952 & 0 & 2 & 10 \\
\hline 15 & 10 & 0 & 5 & 0 & 5 & 10 \\
\hline 18 & 10 & 0 & 8 & 0 & 8 & 10 \\
\hline 21 & 10 & 1 & 10 & 1 & 10 & 10 \\
\hline 24 & 10 & 4 & 10 & 4 & 10 & 10 \\
\hline 27 & 10 & 7 & 10 & 7 & 10 & 10 \\
\hline
\end{tabular}

$b=1$ for $P_{T}=3$ and $P_{T}=6$, which is in compliance with Corollary 4.5. In addition, the individual target CRLBs corresponding to the optimal power vector for $\bar{P}_{T}=27$ and $\lambda=$ 0.2 in Table III are obtained as [0.6498 0.6498 0.8076 0.7547 2.0239]. Consider the PMF $v[i]=\lambda g[i]+(1-\lambda) f[i]$ where $\lambda=0.2, f[i]=0.5$ for $i=1,2$ and $f[i]=0$ otherwise. Then, the solution of Scheme 1 in (18) for $v[i]$ is given by $\boldsymbol{p}_{*}^{J}=$ $[10710]^{T}$, which satisfies (23) in Proposition 4.1 and constitutes a solution of the restricted scheme in (22) for $g[i]$, in compliance with Proposition 4.1. Also, it is noted that $f[i]$ contains 2 point masses (i.e., the solution of Scheme 2 is determined by 2 target nodes), which is in accordance with Proposition 4.6. Moreover, choosing the design parameter of the problem in (21) as $\alpha=\min _{i \in\left\{1,2, \ldots, N_{T}\right\}} C_{i}\left(\boldsymbol{p}_{*}^{J}\right)=0.6498$, the solution of (21) is given by $\boldsymbol{p}_{*}^{J}$, which agrees with Corollary 4.2.

2) Alternative Scheme: The alternative scheme is illustrated in Fig. 11 for the scenario in Fig. 7. The figure shows the number of disabled target nodes versus $t$ for various values of normalized total power $\bar{P}_{T}$. For both $\bar{P}_{T}=14$ and $\bar{P}_{T}=17$, it is calculated that $\Lambda_{4}=\{1,3,4,5\}, \Lambda_{3}=\{3,4,5\}, \Lambda_{2}=\{3,5\}$ and $\Lambda_{1}=\{5\}$. Based on the above results and those presented in Section VII-A2, it should be noted that whether the relation $\Lambda_{k} \subset \Lambda_{k+1}$ holds or not depends on the total power constraint and the specific network configuration, which determines the

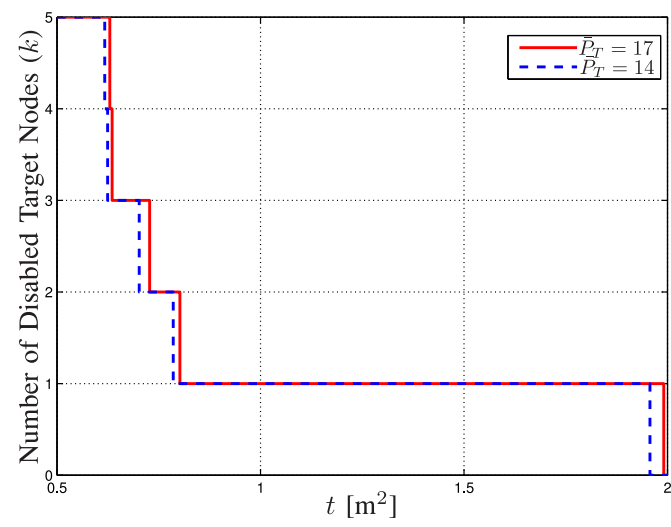

Fig. 11. Alternative schemes (for $\bar{P}_{T}=14$ and $\bar{P}_{T}=17$ ) versus $t$ for the scenario in Fig. 7.

TABLE IV

PARAMETER $t_{k}$ AND ALLOCATED POWERS TO JAMMER NODES FOR THE ALTERNATIVE SCHEME FOR THE SCENARIO IN FIG. 7

\begin{tabular}{|l|llll|llll|}
\hline \multirow{2}{*}{$k$} & \multicolumn{3}{|c|}{$P_{T}=14$} & \multicolumn{4}{c|}{$P_{T}=17$} \\
\cline { 2 - 9 } & $t_{k}$ & $P_{1}^{J}$ & $P_{2}^{J}$ & $P_{3}^{J}$ & $t_{k}$ & $P_{1}^{J}$ & $P_{2}^{J}$ & $P_{3}^{J}$ \\
\hline 5 & 0.6172 & 10 & 2.7504 & 1.2496 & 0.6298 & 10 & 7 & 0 \\
\hline 4 & 0.6244 & 10 & 1.4173 & 2.5827 & 0.6355 & 10 & 6.2243 & 0.7757 \\
\hline 3 & 0.7020 & 0 & 4.8846 & 9.1154 & 0.7276 & 0 & 7 & 10 \\
\hline 2 & 0.7858 & 1.7376 & 10 & 2.2624 & 0.8021 & 2.9458 & 10 & 4.0542 \\
\hline 1 & 1.9571 & 0 & 4 & 10 & 1.9909 & 0 & 7 & 10 \\
\hline
\end{tabular}

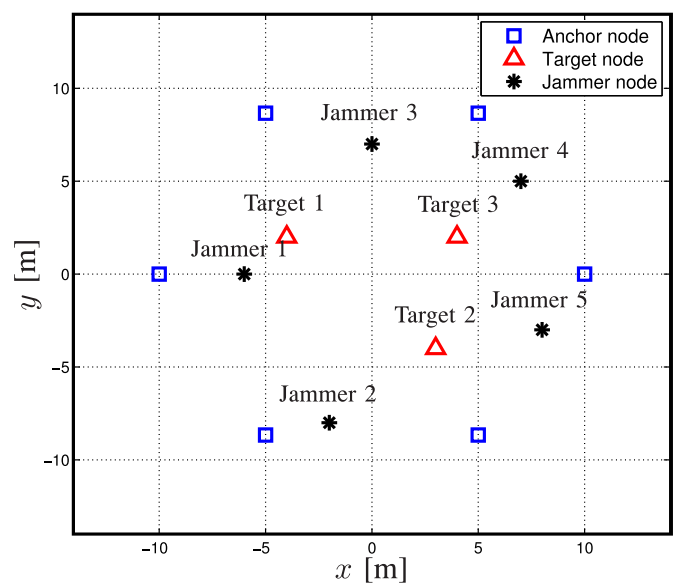

Fig. 12. The network considered in the simulations, where the anchor node positions are $[-100],[-5-5 \sqrt{3}],[-55 \sqrt{3}],[55 \sqrt{3}],[5-5 \sqrt{3}]$, and $[100] \mathrm{m}$., the target node positions are $[-42],[3-4]$ and $[42] \mathrm{m}$., and the jammer node positions are $[-60],[-2-8],[07],[75]$ and $[8-3] \mathrm{m}$.

individual target CRLBs and thus the subnetwork that leads to the maximum max-min CRLB (see Definition 6.1). In addition, Table IV illustrates the optimal power allocation strategies for the alternative schemes along with the corresponding values of $t_{k}$ for various values of $\bar{P}_{T}$ for the scenario in Fig. 7.

\section{Scenario 3: Sparsity of the Optimal Solution}

In the final example, the number of jammer nodes that are effective in the solution of the restricted scheme is investigated for different normalized power levels. A network consisting of 


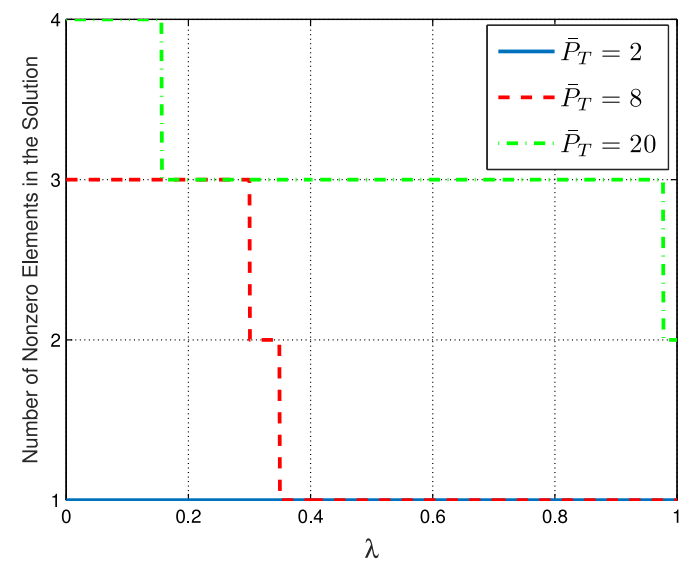

Fig. 13. Number of nonzero elements in the optimal solution of the restricted scheme for various values of $\bar{P}_{T}$ versus $\lambda$ for the scenario in Fig. 12.

six anchor nodes, three target nodes, and five jammer nodes is considered, where the network configuration is shown in Fig. 12. The target significance levels are set as $g[1]=0.5, g[2]=0.3$, and $g[3]=0.2$. In Fig. 13, the number of nonzero elements in the optimal jammer power vector corresponding to the restricted scheme against $\lambda$ is illustrated for various values of $\bar{P}_{T}$. As seen from Fig. 13, the optimal solution contains at most $N_{T}=3$ jammer nodes for $\bar{P}_{T}=2$ and $\bar{P}_{T}=8$, which is in compliance with Proposition 4.6 since the peak power constraint $\left(P_{\ell}^{\text {peak }}=\right.$ $10, \forall \ell)$ is not effective for $\bar{P}_{T}=2$ and $\bar{P}_{T}=8$. However, when the peak power constraint is incorporated by setting $\bar{P}_{T}=20$, the solution of the restricted scheme is determined by more than three jammer nodes for a certain range of values of $\lambda$. Therefore, it can be concluded that the optimal jammer power vector is sparse for sufficiently low values of $\bar{P}_{T}$ (i.e., when the peak power constraint becomes ineffective).

\section{CONCLUDING REMARKS}

In this paper, the restricted scheme has been proposed to investigate optimal power allocation strategies for jammer nodes in a wireless localization system. The restricted scheme aims to maximize the average CRLB of target nodes while keeping the minimum CRLB above a predefined level under average and peak jammer power constraints. Through theoretical analyses, the restricted scheme has been shown to establish a trade-off between the two extreme cases - maximization of the average and the minimum CRLBs. Then, a closed-form solution has been derived under certain conditions on the design parameter that adjusts the trade-off. In addition, it has been proved that the average CRLB of the restricted scheme is a strictly decreasing and concave function of the constraint on the minimum CRLB level. Furthermore, it has been demonstrated that the optimal solution of the restricted scheme involves at most $N_{T}$ jammer nodes, where $N_{T}$ is the number of target nodes, and that the optimal solution of the minimum CRLB maximization scheme corresponds to at most $N_{J}$ target nodes, where $N_{J}$ is the number of jammer nodes. In order to cover more generic scenarios, an extension to the restricted scheme has been proposed, where target nodes are assigned to subsets depending on their significance levels and the tolerable CRLB level is set differently for each subset in the formulation. In addition to the restricted scheme, an alternative scheme has been proposed, where the number of disabled target nodes is considered as the optimization criterion. Extensive simulations carried out for various wireless network configurations have exemplified the theoretical results and illustrated the trade-off characteristics of the restricted scheme.

Theoretical and numerical results obtained in this study provide important insights into the design of efficient jamming algorithms in practical scenarios. For instance, the sparsity property of the optimal jammer power vector leads to a significant simplification of the solution for wireless networks containing fewer target nodes than jammer nodes. In addition, the proposed power allocation criterion puts forward a new and generalized framework for power/resource optimization and thus can be employed in different problems, such as the problem of power allocation among anchor nodes for CRLB minimization of target nodes, where the objective is to minimize the average CRLB while keeping the maximum CRLB below a predefined threshold.

\section{APPENDIX}

\section{A. Average CRLB Minimization Property of Least-Favorable $P M F$}

Let $g[i]$ and $\tilde{g}[i]$ denote, respectively, the least-favorable PMF and any valid PMF defined over the set $\left\{1,2, \ldots, N_{T}\right\}$. Also, let $\boldsymbol{p}_{*}^{J}$ and $\boldsymbol{p}_{+}^{J}$ represent the solutions of Scheme 1 for $g[i]$ and $\tilde{g}[i]$, respectively. Then,

$$
\begin{aligned}
\sum_{i=1}^{N_{T}} g[i] C_{i}\left(\boldsymbol{p}_{*}^{J}\right)=\min _{i \in\left\{1,2, \ldots, N_{T}\right\}} C_{i}\left(\boldsymbol{p}_{*}^{J}\right) & \leq \sum_{i=1}^{N_{T}} \tilde{g}[i] C_{i}\left(\boldsymbol{p}_{*}^{J}\right) \\
& \leq \sum_{i=1}^{N_{T}} \tilde{g}[i] C_{i}\left(\boldsymbol{p}_{+}^{J}\right)
\end{aligned}
$$

where the equality is due to (19), the first inequality is by definition, and the last inequality follows from the fact that $\boldsymbol{p}_{+}^{J}$ is the solution of Scheme 1 for $\tilde{g}[i]$. The overall inequality in (47) indicates that the least-favorable PMF minimizes the average CRLB among all PMFs.

\section{B. Equivalence of Scheme 1 and Scheme 2}

Assume that $g[i]$ is the least-favorable PMF and that $\boldsymbol{p}_{*}^{J}$ is the solution of Scheme 1 for $g[i]$. Then,

$$
\begin{array}{r}
\min _{i \in\left\{1,2, \ldots, N_{T}\right\}} C_{i}\left(\boldsymbol{p}_{*}^{J}\right)=\sum_{i=1}^{N_{T}} g[i] C_{i}\left(\boldsymbol{p}_{*}^{J}\right) \geq \sum_{i=1}^{N_{T}} g[i] C_{i}\left(\boldsymbol{p}^{J}\right) \\
\geq \min _{i \in\left\{1,2, \ldots, N_{T}\right\}} C_{i}\left(\boldsymbol{p}^{J}\right)
\end{array}
$$

for any power vector $\boldsymbol{p}^{J}$ satisfying the total and peak power constraints. Hence, $\boldsymbol{p}_{*}^{J}$ is also the solution of Scheme 2. 


\section{Proof of Proposition 4.3}

The steps of the proof are similar to those in [25, Theorem 2]. Namely,

$$
\begin{aligned}
& \sum_{i=1}^{N_{T}} v[i] C_{i}\left(\boldsymbol{p}_{*}^{J}\right) \\
& =\lambda \sum_{i=1}^{N_{T}} g[i] C_{i}\left(\boldsymbol{p}_{*}^{J}\right)+(1-\lambda) \sum_{i=1}^{N_{T}} f[i] C_{i}\left(\boldsymbol{p}_{*}^{J}\right) \\
& =\lambda C_{\mathrm{avg}}\left(\boldsymbol{p}_{*}^{J}\right)+(1-\lambda) \min _{i \in\left\{1,2, \ldots, N_{T}\right\}} C_{i}\left(\boldsymbol{p}_{*}^{J}\right) \\
& \leq \tilde{\lambda} C_{\mathrm{avg}}\left(\boldsymbol{p}_{*}^{J}\right)+(1-\tilde{\lambda}) \min _{i \in\left\{1,2, \ldots, N_{T}\right\}} C_{i}\left(\boldsymbol{p}_{*}^{J}\right) \\
& \leq \tilde{\lambda} \sum_{i=1}^{N_{T}} g[i] C_{i}\left(\boldsymbol{p}_{*}^{J}\right)+(1-\tilde{\lambda}) \sum_{i=1}^{N_{T}} \tilde{f}[i] C_{i}\left(\boldsymbol{p}_{*}^{J}\right) \\
& =\sum_{i=1}^{N_{T}}(\tilde{\lambda} g[i]+(1-\tilde{\lambda}) \tilde{f}[i]) C_{i}\left(\boldsymbol{p}_{*}^{J}\right) \\
& =\sum_{i=1}^{N_{T}} \tilde{v}[i] C_{i}\left(\boldsymbol{p}_{*}^{J}\right) \leq \sum_{i=1}^{N_{T}} \tilde{v}[i] C_{i}\left(\boldsymbol{p}_{+}^{J}\right)
\end{aligned}
$$

where (50) follows from (23), (51) follows from the inequality $\tilde{\lambda} \geq \lambda$ and that $C_{\mathrm{avg}}\left(\boldsymbol{p}_{*}^{J}\right) \geq \min _{i \in\left\{1,2, \ldots, N_{T}\right\}} C_{i}\left(\boldsymbol{p}_{*}^{J}\right)$, (52) is due to the relation between the minimum and the average operators, and (54) follows from that $\boldsymbol{p}_{+}^{J}$ is the solution of Scheme 1 for $\tilde{v}[i]$. Hence, (30) in Proposition 4.3 is obtained.

\section{Proof of Proposition 4.4}

The proof is constructed based on similar arguments to those in [25, Lemma]. First, the concavity of $C_{\mathrm{avg}}\left(\boldsymbol{p}_{r}^{J}(\alpha)\right)$ is proved. From the definition of the restricted scheme in (21), it can be concluded that $C_{\mathrm{avg}}\left(\boldsymbol{p}_{r}^{J}(\alpha)\right)$ is a non-increasing function of $\alpha$. The new power vector $\boldsymbol{p}^{J}$ is defined as a randomization of two power vectors corresponding to the solutions of the restricted scheme for $\alpha_{1}$ and $\alpha_{2}$ :

$$
\boldsymbol{p}^{J} \triangleq \kappa \boldsymbol{p}_{r}^{J}\left(\alpha_{1}\right)+(1-\kappa) \boldsymbol{p}_{r}^{J}\left(\alpha_{2}\right)
$$

where $0 \leq \alpha_{1} \leq \alpha_{2} \leq \bar{\alpha}$ and $0<\kappa<1$. From the definition of $\boldsymbol{p}^{J}$, it is clear that $\boldsymbol{p}^{\bar{J}}$ satisfies both the total and peak power constraints. The following relation on the average CRLB corresponding to $\boldsymbol{p}^{J}$ is obtained via (15) and (55):

$$
C_{\mathrm{avg}}\left(\boldsymbol{p}^{J}\right)=\kappa C_{\mathrm{avg}}\left(\boldsymbol{p}_{r}^{J}\left(\alpha_{1}\right)\right)+(1-\kappa) C_{\mathrm{avg}}\left(\boldsymbol{p}_{r}^{J}\left(\alpha_{2}\right)\right) .
$$

Similarly, for the minimum CRLB corresponding to $\boldsymbol{p}^{J}$,

$$
\begin{aligned}
\min _{i \in T} C_{i}\left(\boldsymbol{p}^{J}\right) & \geq \kappa \min _{i \in T} C_{i}\left(\boldsymbol{p}_{r}^{J}\left(\alpha_{1}\right)\right)+(1-\kappa) \min _{i \in T} C_{i}\left(\boldsymbol{p}_{r}^{J}\left(\alpha_{2}\right)\right) \\
& \geq \kappa \alpha_{1}+(1-\kappa) \alpha_{2},
\end{aligned}
$$

where $T=\left\{1,2, \ldots, N_{T}\right\}$. Let $\alpha=\min _{i \in T} C_{i}\left(\boldsymbol{p}^{J}\right)$ and $\alpha^{*}=$ $\kappa \alpha_{1}+(1-\kappa) \alpha_{2}$, then $\alpha \geq \alpha^{*}$ is concluded from (57). This results in

$$
\begin{aligned}
C_{\mathrm{avg}}\left(\boldsymbol{p}_{r}^{J}\left(\alpha^{*}\right)\right) & \geq C_{\mathrm{avg}}\left(\boldsymbol{p}_{r}^{J}(\alpha)\right) \\
& \geq C_{\mathrm{avg}}\left(\boldsymbol{p}^{J}\right) \\
& =\kappa C_{\mathrm{avg}}\left(\boldsymbol{p}_{r}^{J}\left(\alpha_{1}\right)\right)+(1-\kappa) C_{\mathrm{avg}}\left(\boldsymbol{p}_{r}^{J}\left(\alpha_{2}\right)\right)
\end{aligned}
$$

which proves the concavity of $C_{\mathrm{avg}}\left(\boldsymbol{p}_{r}^{J}(\alpha)\right)$, where (58) follows from the non-increasing property of $C_{\mathrm{avg}}\left(\boldsymbol{p}_{r}^{J}(\alpha)\right)$, (59) is due to the definition of the restricted scheme with $\alpha=$ $\min _{i \in T} C_{i}\left(\boldsymbol{p}^{J}\right)$, and (60) follows from (56).

Next, the strictly decreasing property of $C_{\mathrm{avg}}\left(\boldsymbol{p}_{r}^{J}(\alpha)\right)$ is proved. To that end, the following equality is first shown to hold for $\alpha \in(\underline{\alpha}, \bar{\alpha})$ :

$$
\min _{i \in T} C_{i}\left(\boldsymbol{p}_{r}^{J}(\alpha)\right)=\alpha
$$

Assume that $\min _{i \in T} C_{i}\left(\boldsymbol{p}_{r}^{J}(\alpha)\right)>\alpha$, and define $\boldsymbol{p}^{J} \triangleq \kappa \boldsymbol{p}_{1}^{J}$ $+(1-\kappa) \boldsymbol{p}_{r}^{J}(\alpha)$, where $0<\kappa<1$ is selected so that $\min _{i \in T} C_{i}\left(\boldsymbol{p}^{J}\right) \geq \alpha$ (it is clear that $\boldsymbol{p}^{J}$ satisfies the average and peak power constraints). The existence of such a $\kappa$ can be proved as follows:

$$
\begin{aligned}
\min _{i \in T} C_{i}\left(\boldsymbol{p}^{J}\right) & =\min _{i \in T}\left(\kappa \boldsymbol{d}_{i}^{T} \boldsymbol{p}_{1}^{J}+(1-\kappa) \boldsymbol{d}_{i}^{T} \boldsymbol{p}_{r}^{J}(\alpha)\right) \\
& \geq \kappa \min _{i \in T} \boldsymbol{d}_{i}^{T} \boldsymbol{p}_{1}^{J}+(1-\kappa) \min _{i \in T} \boldsymbol{d}_{i}^{T} \boldsymbol{p}_{r}^{J}(\alpha) \\
& =\kappa \min _{i \in T} C_{i}\left(\boldsymbol{p}_{1}^{J}\right)+(1-\kappa) \min _{i \in T} C_{i}\left(\boldsymbol{p}_{r}^{J}(\alpha)\right)
\end{aligned}
$$

where (62) and (64) follows from (15). Since $\min _{i \in T} C_{i}\left(\boldsymbol{p}_{1}^{J}\right)=$ $\underline{\alpha}<\alpha$ and $\min _{i \in T} C_{i}\left(\boldsymbol{p}_{r}^{J}(\alpha)\right)>\alpha$, there exists $\kappa \in(0,1)$ such that the expression in (64) is equal to $\alpha$. Hence, the proof for the existence of $\kappa$ is completed. From the definition of Scheme 1 , the average CRLB corresponding to $\boldsymbol{p}_{1}^{J}$ is larger than that corresponding to $\boldsymbol{p}_{r}^{J}(\alpha)$ for $\alpha \in(\underline{\alpha}, \bar{\alpha})$, which implies that $C_{\mathrm{avg}}\left(\boldsymbol{p}^{J}\right)>C_{\mathrm{avg}}\left(\boldsymbol{p}_{r}^{J}(\alpha)\right)$, leading to a contradiction with the definition of the restricted scheme. (Note that $\boldsymbol{p}^{J}$ satisfies the constraints in (21).) Thus, (61) must hold. Finally, let $\underline{\alpha}<\alpha_{1}<$ $\alpha_{2}<\bar{\alpha}$, and assume that $C_{\mathrm{avg}}\left(\boldsymbol{p}_{r}^{J}\left(\alpha_{1}\right)\right)=C_{\mathrm{avg}}\left(\boldsymbol{p}_{r}^{J}\left(\alpha_{2}\right)\right)$. Then, $\boldsymbol{p}_{r}^{J}\left(\alpha_{1}\right)$ is also the solution of the restricted scheme for $\alpha_{2}$, which requires that $\min _{i \in T} C_{i}\left(\boldsymbol{p}_{r}^{J}\left(\alpha_{2}\right)\right)=\alpha_{1}$, which in turn contradicts with the fact that $\min _{i \in T} C_{i}\left(\boldsymbol{p}_{r}^{J}\left(\alpha_{2}\right)\right)=\alpha_{2}$. Therefore, $C_{\mathrm{avg}}\left(\boldsymbol{p}_{r}^{J}\left(\alpha_{1}\right)\right)>C_{\mathrm{avg}}\left(\boldsymbol{p}_{r}^{J}\left(\alpha_{2}\right)\right)$ must hold.

\section{REFERENCES}

[1] R. Zekavat and R. M. Buehrer, Handbook of Position Location: Theory, Practice and Advances. New York, NY, USA: Wiley, 2011.

[2] J. Figueiras and S. Frattasi, Mobile Positioning and Tracking: From Conventional to Cooperative Techniques. Chichester, U.K.: Wiley, 2010.

[3] Z. Sahinoglu, S. Gezici, and I. Guvenc, Ultra-Wideband Positioning Sys tems: Theoretical Limits, Ranging Algorithms, and Protocols. New York, NY, USA: Cambridge Univ. Press, 2008.

[4] S. Gezici et al., "Localization via ultra-wideband radios: A look at positioning aspects for future sensor networks," IEEE Signal Process. Mag., vol. 22, no. 4, pp. 70-84, Jul. 2005.

[5] S. Gezici, "A survey on wireless position estimation," Wireless Pers. Com mun., vol. 44, no. 3, pp. 263-282, Feb. 2008.

[6] Y. Shen and M. Z. Win, "Fundamental limits of wideband localizationPart I: A general framework," IEEE Trans. Inf. Theory, vol. 56, no. 10, pp. 4956-4980, Oct. 2010 
[7] K. Grover, A. Lim, and Q. Yang, "Jamming and anti-jamming techniques in wireless networks: A survey," Int. J. Ad Hoc Ubiquitous Comput. vol. 17, no. 4, pp. 197-215, Dec. 2014. [Online]. Available: http://dx.doi. org/10.1504/IJAHUC.2014.066419

[8] H. Hu and N. Wei, "A study of GPS jamming and anti-jamming," in Proc 2nd Int. Conf. Power Electron. Intell. Transp. Syst., Dec. 2009, vol. 1, pp. 388-391.

[9] D. Lu, R. Wu, and H. Liu, "Global positioning system anti-jamming algorithm based on period repetitive CLEAN," IET Radar, Sonar Navig. vol. 7, no. 2, pp. 1640-169, Feb. 2013.

[10] Y. Zhang and M. Amin, "Anti-jamming GPS receiver with reduced phase distortions," IEEE Signal Process. Lett., vol. 19, no. 10, pp. 635-638, Oct. 2012.

[11] C. W. Commander, P. M. Pardalos, V. Ryabchenko, O. Shylo, S. Uryasev, and G. Zrazhevsky, "Jamming communication networks under complete uncertainty," Optim. Lett., vol. 2, no. 1, pp. 53-70, 2008

[12] M. Li, I. Koutsopoulos, and R. Poovendran, "Optimal jamming attack strategies and network defense policies in wireless sensor networks," IEEE Trans. Mobile Comput., vol. 9, no. 8, pp. 1119-1133, Aug. 2010.

[13] S. Gezici, M. R. Gholami, S. Bayram, and M. Jansson, "Jamming of wireless localization systems," IEEE Trans. Commun., vol. 64, no. 6, pp. 2660-2676, Jun. 2016.

[14] S. Gezici, S. Bayram, M. N. Kurt, and M. R. Gholami, "Optimal jammer placement in wireless localization systems," IEEE Trans. Signal Process. vol. 64, no. 17, pp. 4534-4549, Sep. 2016.

[15] W. W.-L. Li, Y. Shen, Y. J. Zhang, and M.Z. Win, "Robust power allocation for energy-efficient location-aware networks," IEEE/ACM Trans. Netw., vol. 21, no. 6, pp. 1918-1930, Dec. 2013.

[16] W. Dai, Y. Shen, and M. Z. Win, "Distributed power allocation for cooperative wireless network localization," IEEE J. Select. Areas Commun., vol. 33, no. 1, pp. 28-40, Jan. 2015.

[17] T. Wang, G. Leus, and L. Huang, "Ranging energy optimization for robust sensor positioning based on semidefinite programming," IEEE Trans. Signal Process., vol. 57, no. 12, pp. 4777-4787, Dec. 2009.

[18] T. Zhang, A. F. Molisch, Y. Shen, Q. Zhang, H. Feng, and M. Z. Win, "Joint power and bandwidth allocation in wireless cooperative localization networks," IEEE Trans. Wireless Commun., vol. 15, no. 10, pp. 6527-6540, Oct. 2016.

[19] T. Zhang, C. Qin, A. F. Molisch, and Q. Zhang, "Joint allocation of spectral and power resources for non-cooperative wireless localization networks," IEEE Trans. Commun., vol. 64, no. 9, pp. 3733-3745, Sep. 2016.

[20] J. Chen, W. Dai, Y. Shen, V. K. N. Lau, and M. Z. Win, "Power management for cooperative localization: A game theoretical approach," IEEE Trans. Signal Process., vol. 64, no. 24, pp. 6517-6532, Dec. 2016.

[21] G. Alirezaei, M. Reyer, and R. Mathar, "Optimum power allocation in sensor networks for passive radar applications," IEEE Trans. Wireless Commun., vol. 13, no. 6, pp. 3222-3231, Jun. 2014.

[22] H. Godrich, A. Petropulu, and H. Poor, "Power allocation strategies for target localization in distributed multiple-radar architectures," IEEE Trans. Signal Process., vol. 59, no. 7, pp. 3226-3240, Jul. 2011

[23] P. Chavali and A. Nehorai, "Scheduling and power allocation in a cognitive radar network for multiple-target tracking," IEEE Trans. Signal Process. vol. 60 , no. 2 , pp. 715-729, Feb. 2012.

[24] E. L. Lehmann, Testing Statistical Hypotheses. 2nd ed. New York, NY, USA: Chapman \& Hall, 1986.

[25] J. L. Hodges Jr., and E. L. Lehmann, "The use of previous experience in reaching statistical decisions," Ann. Math. Statist., vol. 23, pp. 396-407, Sep. 1952

[26] M. K. Simon, J. K. Omura, R. A. Scholtz, and B. K. Levitt, Spread Spectrum Communications, vol. 1, Rockville, MD, USA: Comput. Sci. Press, 1985

[27] M. Weiss and S. C. Schwartz, "On optimal minimax jamming and detection of radar signals," IEEE Trans. Aerosp. Electron. Syst., vol. AES-21, no. 3, pp. 385-393, May 1985.

[28] R. J. McEliece and W. E. Stark, "An information theoretic study of communication in the presence of jamming," in Proc. IEEE Int. Conf. Commun., 1981, vol. 3, pp. 45.3.1-45.3.5.

[29] Y. Qi and H. Kobayashi, "Cramér-Rao lower bound for geolocation in non-line-of-sight environment," in Proc. IEEE Int. Conf. Acoust., Speech, Signal Process., May 2002, vol. 3, pp. III-2473-III-2476.

[30] Y. Qi, H. Kobayashi, and H. Suda, "Analysis of wireless geolocation in a non-line-of-sight environment," IEEE Trans. Wireless Commun., vol. 5 no. 3, pp. 672-681, Mar. 2006.
[31] A. Mallat, S. Gezici, D. Dardari, C. Craeye, and L. Vandendorpe, "Statistics of the MLE and approximate upper and lower bounds - Part I: Application to TOA estimation," IEEE Trans. Signal Process., vol. 62, no. 21, pp. 5663-5676, Nov. 2014

[32] Y. Shen, W. Dai, and M. Win, "Power optimization for network localization," IEEE/ACM Trans. Netw., vol. 22, no. 4, pp. 1337-1350, Aug. 2014.

[33] A. Wald, Statistical Decision Functions. New York, NY, USA: Wiley, 1950

[34] J. v. Neumann, "Zur Theorie der Gesellschaftsspiele," Mathematische Annalen, vol. 100, pp. 295-320, 1928. [Online]. Available: http://eudml. org/doc/159291

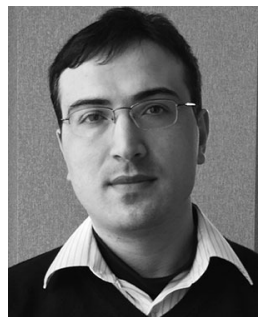

Suat Bayram received the B.S. degree from Middle East Technical University, Ankara, Turkey, in 2007, and the M.S. and the Ph.D. degrees from Bilkent University, Ankara, Turkey, in 2009 and 2011, respectively. In 2015, he founded his own technology company. He is currently a Senior Engineer in PaneraTech, Chantilly, VA, USA. His research interests include statistical signal processing, radar signal processing, and communications.

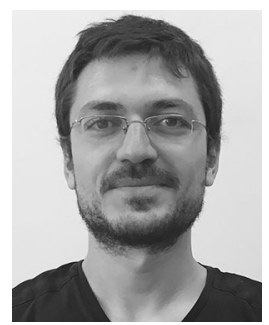

Musa Furkan Keskin received the B.S. and M.S degrees, in 2010 and 2012, respectively, from the Department of Electrical and Electronics Engineering, Bilkent University, Ankara, Turkey, where he is currently working toward the Ph.D. degree. His research interests include signal processing, wireless localization, and visible light communications.

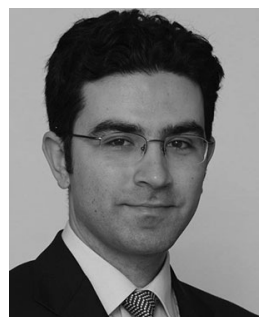

Sinan Gezici (S'03-M'06-SM'11) received the B.S degree from Bilkent University, Ankara, Turkey, in 2001, and the Ph.D. degree in electrical engineering from Princeton University, Princeton, NJ, USA, in 2006. From 2006 to 2007, he was in Mitsubish Electric Research Laboratories, Cambridge, MA, USA. Since 2007, he has been in the Department of Electrical and Electronics Engineering, Bilkent University, where he is currently a Professor. His research interests include detection and estimation theory, wireless communications, localization systems, and visible light communications. Among his publications in these areas is the book Ultra-Wideband Positioning Systems: Theoretical Limits, Ranging Algorithms, and Protocols (Cambridge University Press, 2008). He is an Associate Editor of the IEEE TRANSACTIONS ON COMMUNICATIONS and IEEE WIRELESS COMMUNICATIONS LETTERS.

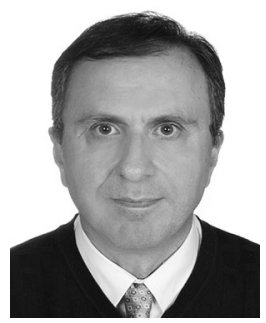

Orhan Arikan was born in Manisa, Turkey, in 1964 He received the B.Sc. degree in electrical and electronics engineering from the Middle East Technica University, Ankara, Turkey, in 1986, and the M.S. and $\mathrm{Ph}$.D. degrees in electrical and computer engineering from the University of Illinois Urbana-Champaign, Champaign, IL, USA, in 1988 and 1990, respectively. Following his graduate studies, he worked for three years as a Research Scientist in Schlumberger-Doll Research Center, Ridgefield, CT, USA. During this time, he was involved in the inverse problems and fusion of multiple modality measurements. In 1993, he joined the Electrical and Electronics Engineering Department, Bilkent University, Ankara, Turkey, where he is currently a Professor and the Chairman. His research interests include statistical signal processing and remote sensing. In 1998, he received the Distinguished Teaching Award of Bilkent University. In 2002, he received the Young Investigator Award in Engineering from Turkish Scientific and Technical Research Foundation. He was the Chairman of IEEE Signal Processing Society, Turkey Section in 1995-1996, and the President of IEEE Turkey Section in 2000-2001. 